\title{
Ethereal Hydroperoxides: Powerful Reagents for \\ S-Oxygenation of Bridging Thiophenolate Functions
}

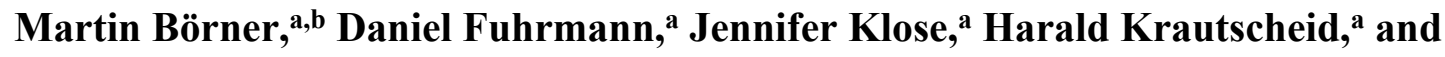 \\ Berthold Kersting ${ }^{a^{*}}$
}

${ }^{a}$ Institut für Anorganische Chemie, Universität Leipzig, Johannisallee 29, 04103 Leipzig,

Germany, E-mail: b.kersting@uni-leipzig.de Fax: +49(0)341-97-36199

${ }^{b}$ Leibniz-Institut für Oberflächenmodifizierung (IOM), Abteilung Funktionale Oberflächen, Permoserstr. 15, D-04318 Leipzig, Germany

\section{Supporting Information}

\section{Contents}

1. Characterization data for $\left[\mathrm{Ni}_{2} \mathrm{~L}^{\mathrm{SO} 2}\left(\mathrm{O}_{2} \mathrm{CPh}\right)\right] \mathrm{ClO}_{4}(2)$ : ESI-MS, calculated and experimental FTIR-spectra (Figures S1-S3).

2. Characterization data for $\left[\mathrm{Ni}_{2} \mathrm{~L}^{\mathrm{SO}}\left(\mathrm{O}_{2} \mathrm{CPh}\right)\right] \mathrm{ClO}_{4}(3)$ : ESI-MS, calculated and experimental FTIR-spectra (Figures S4-S6), Overlay of FTIR spectra for 1-3 (Fig. S7).

3. Detection of a sulfenate intermediate (Fig. S8).

4. Variable temperature magnetic susceptibility measurements for $\mathbf{1}$ and $\mathbf{2}$ (Table S1, S2).

5. DFT calculated geometries for $\mathbf{2}$ (coordinates Table S3, molecule plot Fig. S9, comparison of exp. and calc. bond lengths, Table S4).

DFT calculated geometries for 3 (coordinates Table S5, molecule plot Fig. S10, comparison of exp. and calc. bond lengths, Table S6).

DFT calculated geometries for pre-2 sulfone form (coordinates Table S7, molecule plot Fig. S11, calc. bond lengths, Table S8).

DFT calculated geometries for pre-2 sulfenate form (coordinates Table S9, molecule plot Fig. S12, calc. bond lengths, Table S10).

DFT calculated geometries for 1 sulfenate form (molecule plot (Fig. S13), comp. calc. and exp. bond lengths (Table S11), coordinates (Table S12).

Plots of $\alpha$ and $\beta$ HOMO molecular orbitals for 1, 2 and pre-2 (Figure S14).

6. Calculation of electronic absorption spectra/transitions for 1 (Figure S15, Table S13), 2 (Figure S16, Table S14), 3 (Figure S17, Table S15) and 5 (Figure S18, Table S16). 


\section{Characterization data for $\left[\mathrm{Ni}_{2} \mathrm{~L}^{\mathrm{SO} 2}\left(\mathrm{O}_{2} \mathrm{CPh}\right)\right] \mathrm{ClO}_{4}(2)$.}
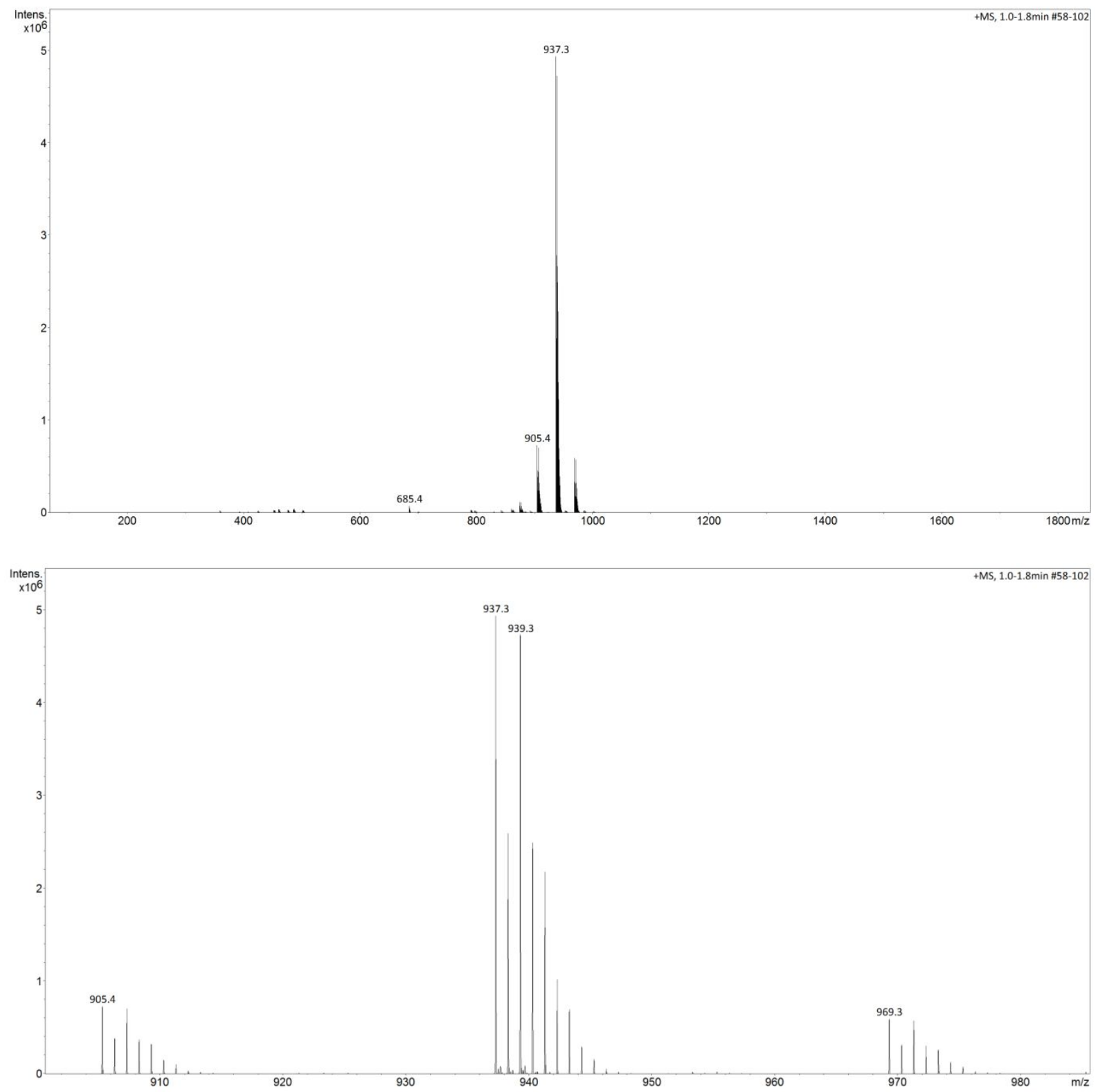

Figure S1. ESI-MS spectrum for $\left[\mathrm{Ni}_{2} \mathrm{~L}^{\mathrm{SO} 2}\left(\mathrm{O}_{2} \mathrm{CPh}\right)\right] \mathrm{ClO}_{4}(2)$. Sample taken from reaction mixture after $72 \mathrm{~h}$ of exposure to $\mathrm{O}_{2}$. The peak at $\mathrm{m} / \mathrm{z}=937.35$ corresponds to the $\left[\mathrm{Ni}_{2} \mathrm{~L}^{\mathrm{SO} 2}\left(\mathrm{O}_{2} \mathrm{CPh}\right)\right]^{+}$cation. The satellite peaks at 905.4 and 969.3 correspond to the cations in $\left[\mathrm{Ni}_{2} \mathrm{~L}^{\mathrm{S}}\left(\mathrm{O}_{2} \mathrm{CPh}\right)\right]^{+}(\mathbf{1})$ and $\left[\mathrm{Ni}_{2} \mathrm{~L}^{\mathrm{SO} 4}\left(\mathrm{O}_{2} \mathrm{CPh}\right)\right]^{+}(\mathbf{3})$. 


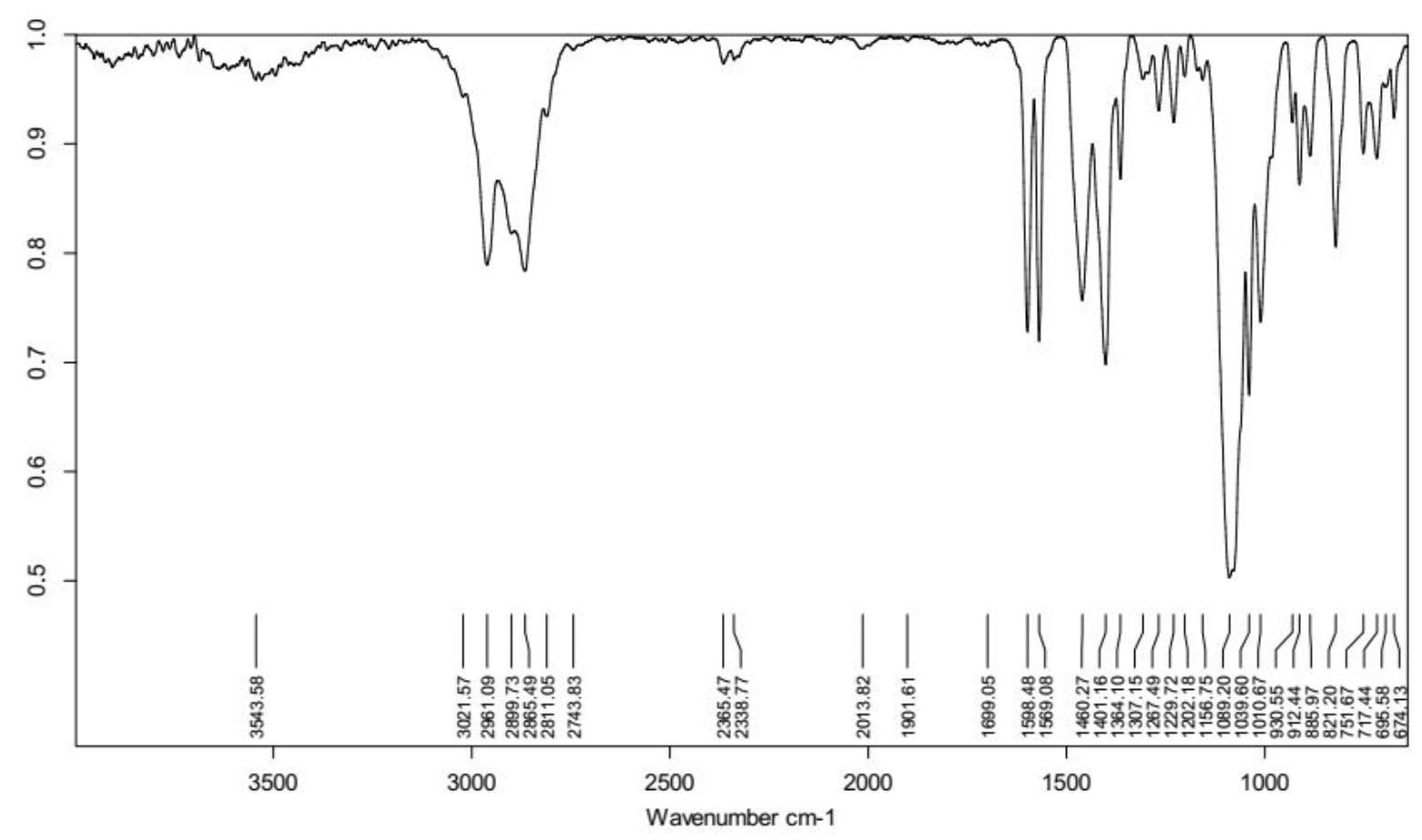

Figure S2. FTIR spectrum of $\left[\mathrm{Ni}_{2} \mathrm{~L}^{\mathrm{SO} 2}\left(\mathrm{O}_{2} \mathrm{CPh}\right)\right] \mathrm{ClO}_{4}(\mathbf{2})(\mathrm{KBr}$ pellet).

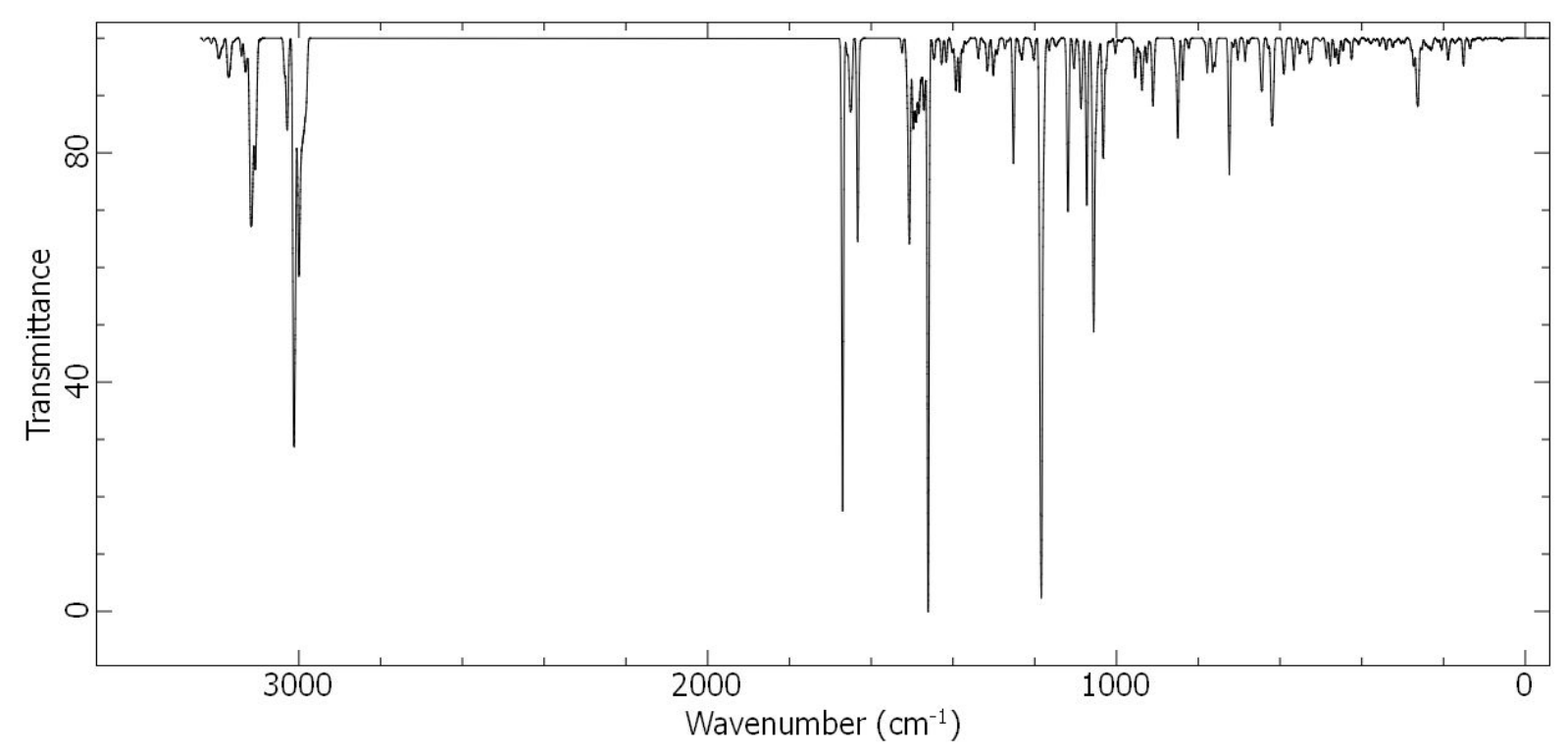

Figure S3. DFT calculated FTIR spectrum of $\left[\mathrm{Ni}_{2} \mathrm{~L}^{\mathrm{SO} 2}\left(\mathrm{O}_{2} \mathrm{CPh}\right)\right]^{+}$(2), unscaled (PBE0/ def2TZVP). 
2. Characterization data for $\left[\mathrm{Ni}_{2} \mathrm{~L}^{\mathrm{SO} 4}\left(\mathrm{O}_{2} \mathrm{CPh}\right)\right] \mathrm{ClO}_{4}(3)$.
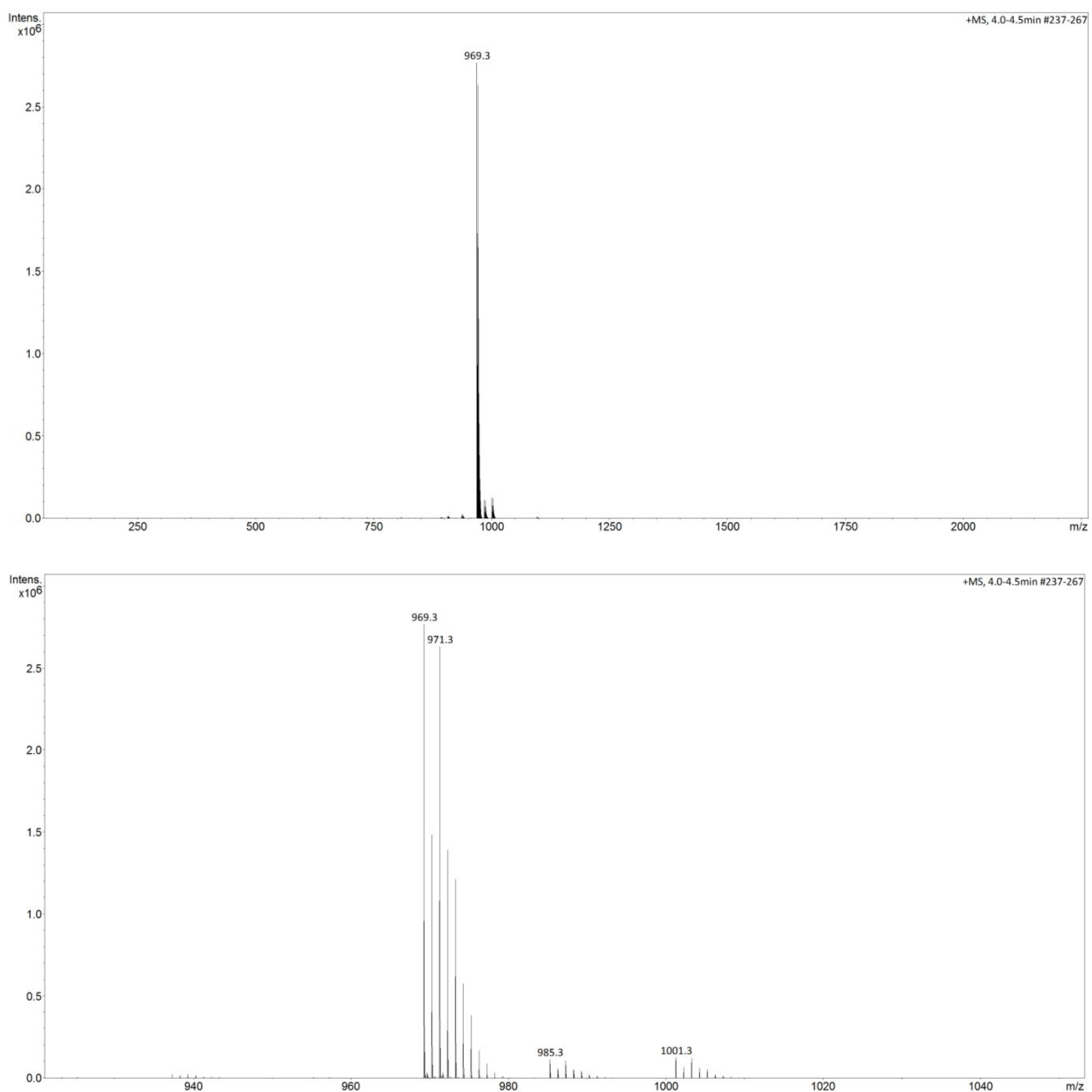

Figure S4. ESI-MS spectrum for $\left[\mathrm{Ni}_{2} \mathrm{~L}^{\mathrm{SO}}\left(\mathrm{O}_{2} \mathrm{CPh}\right)\right] \mathrm{ClO}_{4}(\mathbf{3})$. Sample taken from reaction mixture after $72 \mathrm{~h}$ of exposure to $\mathrm{O}_{2}$. The main peak at $m / z=969.3$ corresponds to the $\left[\mathrm{Ni}_{2} \mathrm{~L}^{\mathrm{SO} 4}\left(\mathrm{O}_{2} \mathrm{CPh}\right)\right]^{+}$cation. The peaks at 985.3 and 1001.3 correspond to higher S-oxygenated species $\left[\mathrm{Ni}_{2} \mathrm{~L}^{\mathrm{SOx}}\left(\mathrm{O}_{2} \mathrm{CPh}\right)\right]^{+}(\mathrm{x}=5,6)$. 


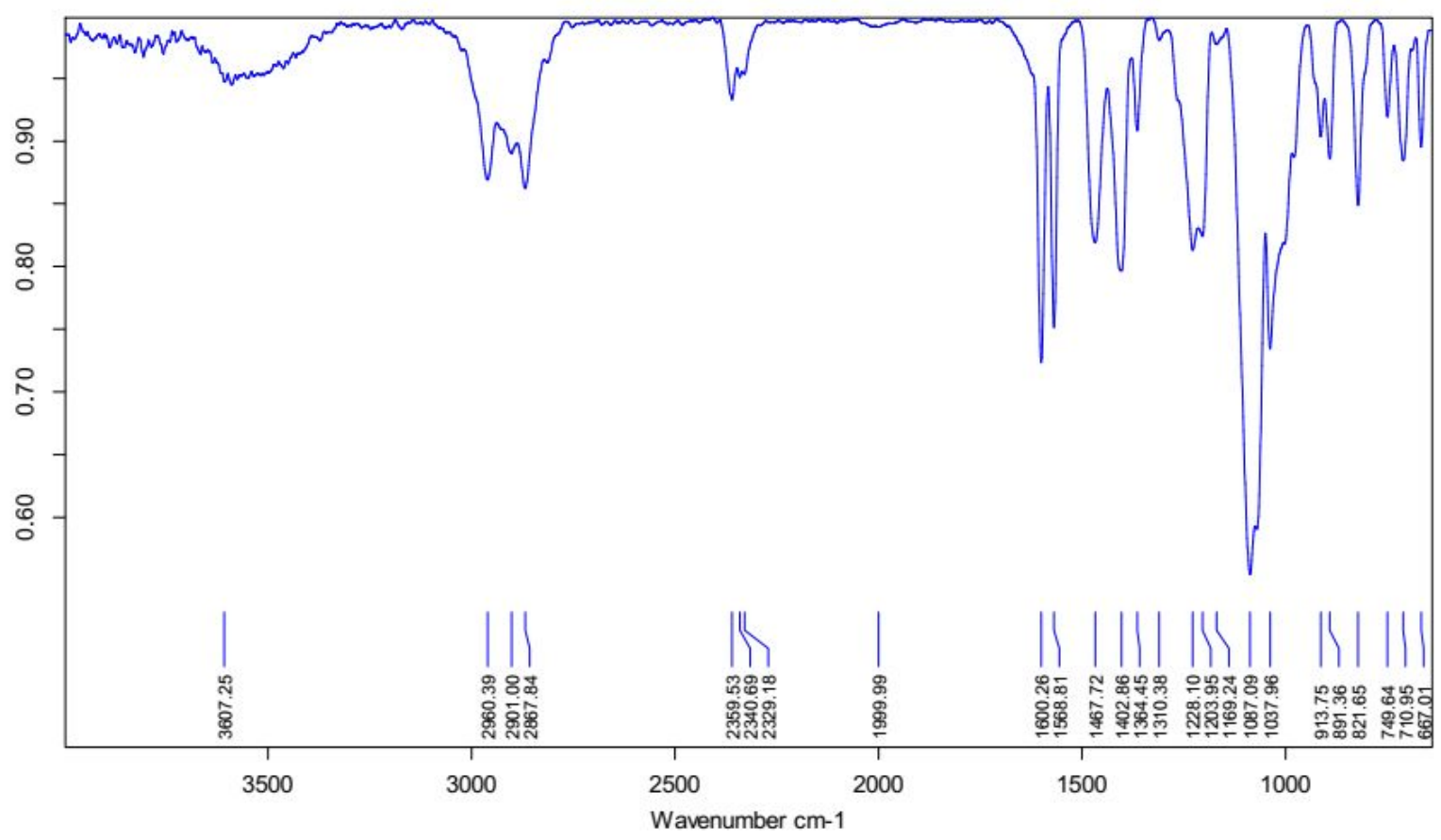

Figure S5. FTIR spectrum of $\left[\mathrm{Ni}_{2} \mathrm{~L}^{\mathrm{SO} 4}\left(\mathrm{O}_{2} \mathrm{CPh}\right)\right] \mathrm{ClO}_{4}(3)(\mathrm{KBr}$ pellet).

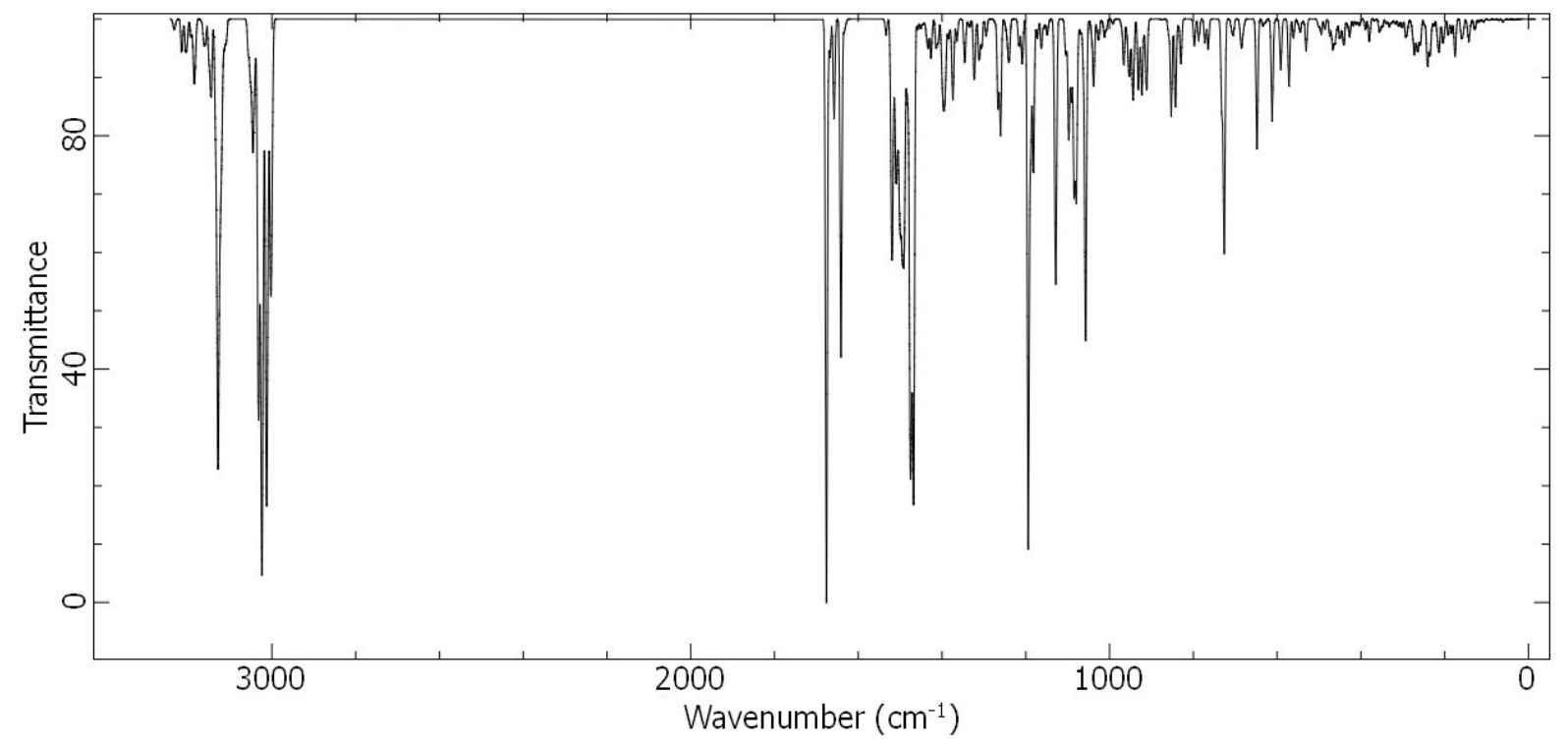

Figure S6. DFT calculated FTIR spectrum of $\left[\mathrm{Ni}_{2} \mathrm{~L}^{\mathrm{SO} 4}\left(\mathrm{O}_{2} \mathrm{CPh}\right)\right]^{+}(\mathbf{3})$, unscaled (PBE0/ def2TZVP) 


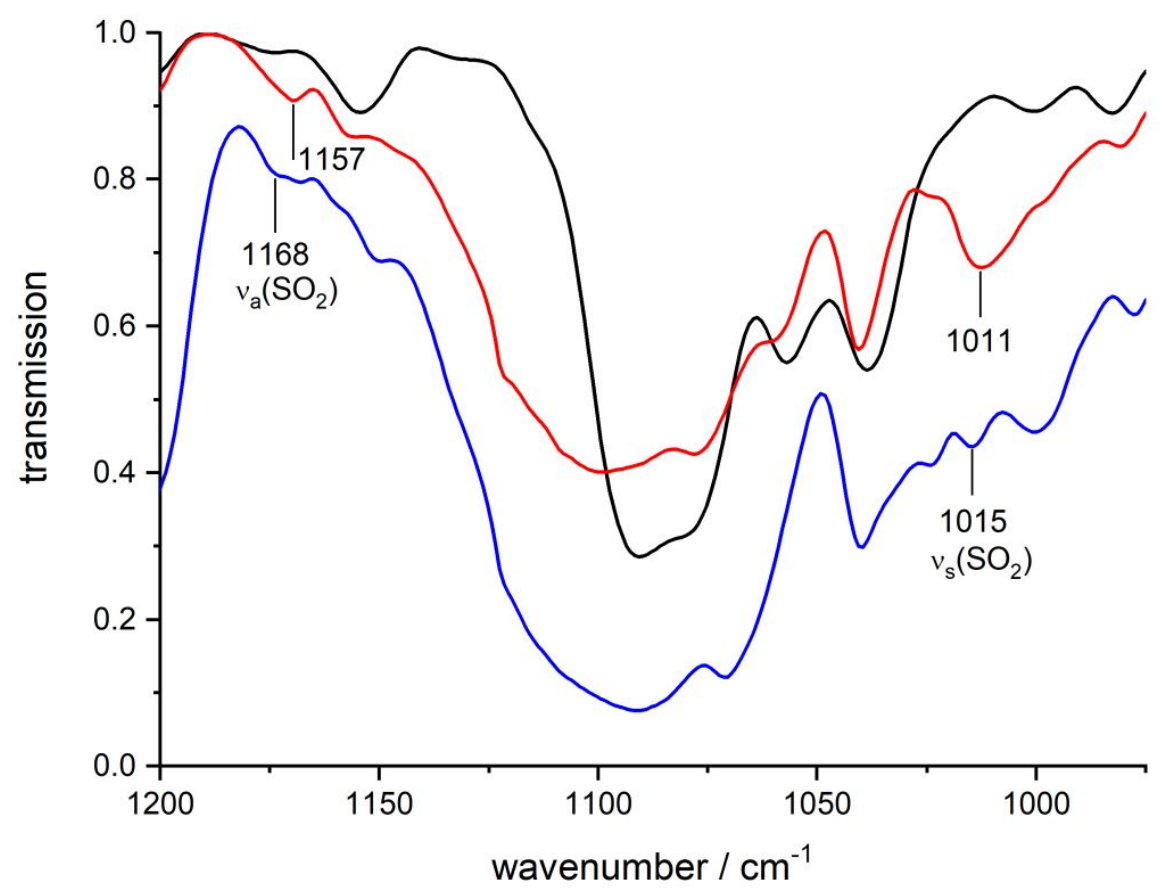

Figure S7. Overlay of the FTIR spectra of $\mathbf{1 ,} 2$ and $\mathbf{3}\left(1200-950 \mathrm{~cm}^{-1}\right.$ region)

\section{Detection of a sulfenate intermediate}

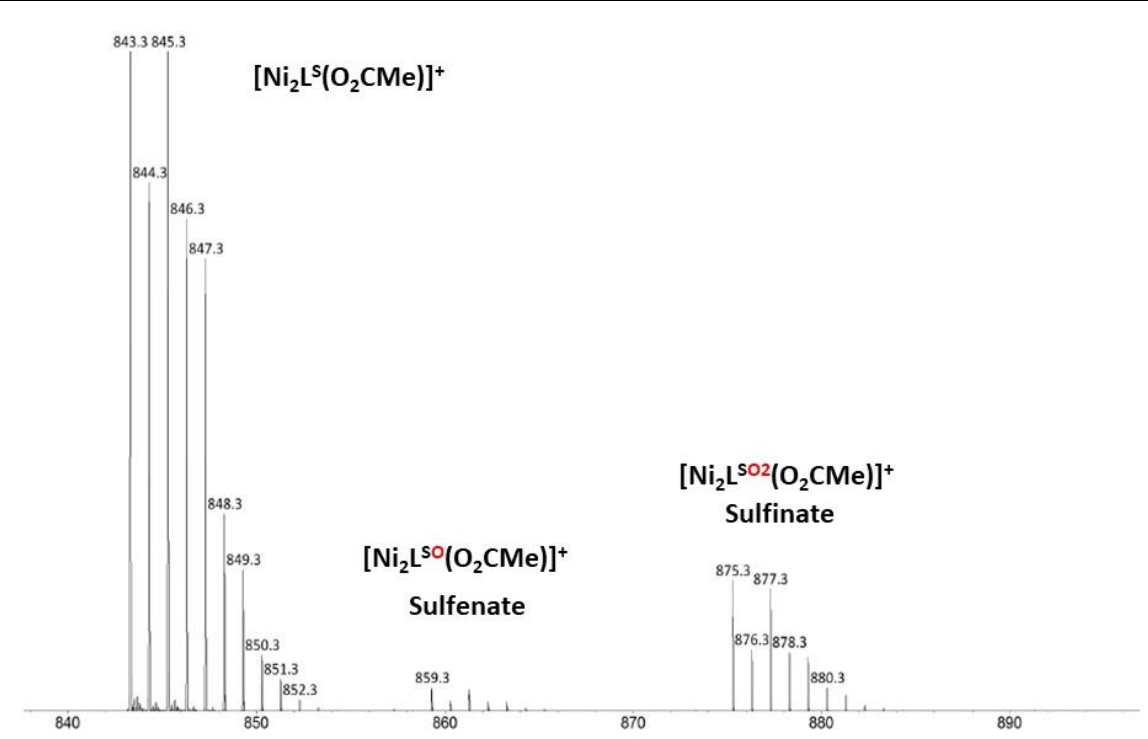

Figure S8. ESI-MS spectrum taken for a solution of $\left[\mathrm{Ni}_{2} \mathrm{~L}^{\mathrm{S}}\left(\mathrm{O}_{2} \mathrm{CMe}\right)\right] \mathrm{ClO}_{4}$ in $\mathrm{MeOH} / \mathrm{MeCN} / 1$,4-dioxane, after stirring for $3 \mathrm{~h}$ with exposure to aerial $\mathrm{O}_{2}$. In this oxygenation experiment, the acetato-bridged complex $\left[\mathrm{Ni}_{2} \mathrm{~L}\left(\mathrm{O}_{2} \mathrm{CMe}\right)\right] \mathrm{ClO}_{4}$ was utilized in place of the benzoate-bridged complex $\left[\mathrm{Ni}_{2} \mathrm{~L}\left(\mathrm{O}_{2} \mathrm{CPh}\right)\right]^{+}(\mathbf{1})$. The peak at $m / z=843.3$ corresponds to the educt $\left[\mathrm{Ni}_{2} \mathrm{~L}^{\mathrm{S}}\left(\mathrm{O}_{2} \mathrm{CMe}\right)\right]^{+}$cation. The peaks at 859.3 and 875.3 correspond to the mono-sulfenate $\left[\mathrm{Ni}_{2} \mathrm{~L}^{\mathrm{SO}}\left(\mathrm{O}_{2} \mathrm{CMe}\right)\right]^{+}$and mono-sulfinate species $\left[\mathrm{Ni}_{2} \mathrm{~L}^{\mathrm{SO}}\left(\mathrm{O}_{2} \mathrm{CMe}\right)\right]^{+}$, respectively. The mono-sulfinate complex, $\left[\mathrm{Ni}_{2} \mathrm{~L}^{\mathrm{SO} 2}\left(\mathrm{O}_{2} \mathrm{CMe}\right)\right]^{+}$has been independently prepared and structurally characterized. These results will be reported elsewhere, but are available from the author upon request. 
4. Variable temperature magnetic susceptibility measurements

Table S1: Experimental and calculated $\chi_{\mathrm{M}} T\left[\mathrm{~cm}^{3} \mathrm{~K} \mathrm{~mol}^{-1}\right]$ and $\mu_{\mathrm{eff}}\left[\mu_{\mathrm{B}}\right]$ values for 2 .

\begin{tabular}{|c|c|c|c|c|}
\hline$T[\mathrm{~K}]$ & $\chi_{M} T_{\exp }$ & $\chi_{M} T_{\text {calc }}$ & $\mu_{\text {eff exp }}$ & $\mu_{\text {eff calc }}$ \\
\hline 330.011 & 2.734 & 2.742 & 4.677 & 4.684 \\
\hline 319.696 & 2.738 & 2.739 & 4.680 & 4.681 \\
\hline 309.453 & 2.731 & 2.736 & 4.675 & 4.678 \\
\hline 299.457 & 2.728 & 2.733 & 4.671 & 4.676 \\
\hline 289.434 & 2.725 & 2.731 & 4.669 & 4.674 \\
\hline 279.487 & 2.724 & 2.729 & 4.668 & 4.672 \\
\hline 269.523 & 2.723 & 2.728 & 4.667 & 4.671 \\
\hline 259.538 & 2.726 & 2.727 & 4.670 & 4.671 \\
\hline 249.557 & 2.725 & 2.727 & 4.669 & 4.670 \\
\hline 239.585 & 2.729 & 2.727 & 4.672 & 4.671 \\
\hline 229.603 & 2.730 & 2.728 & 4.673 & 4.672 \\
\hline 219.666 & 2.735 & 2.731 & 4.677 & 4.674 \\
\hline 209.662 & 2.738 & 2.734 & 4.680 & 4.677 \\
\hline 199.729 & 2.743 & 2.738 & 4.684 & 4.680 \\
\hline 189.767 & 2.750 & 2.743 & 4.690 & 4.685 \\
\hline 179.783 & 2.756 & 2.750 & 4.696 & 4.691 \\
\hline 169.830 & 2.764 & 2.759 & 4.703 & 4.698 \\
\hline 159.853 & 2.775 & 2.769 & 4.712 & 4.707 \\
\hline 149.875 & 2.788 & 2.782 & 4.723 & 4.717 \\
\hline 139.905 & 2.804 & 2.797 & 4.736 & 4.730 \\
\hline 129.920 & 2.823 & 2.815 & 4.752 & 4.746 \\
\hline 119.926 & 2.844 & 2.837 & 4.770 & 4.764 \\
\hline 109.941 & 2.869 & 2.863 & 4.790 & 4.786 \\
\hline 99.991 & 2.898 & 2.894 & 4.815 & 4.812 \\
\hline 89.976 & 2.934 & 2.931 & 4.845 & 4.843 \\
\hline 80.015 & 2.975 & 2.976 & 4.878 & 4.879 \\
\hline 70.044 & 3.022 & 3.029 & 4.917 & 4.922 \\
\hline 60.064 & 3.080 & 3.091 & 4.963 & 4.973 \\
\hline 50.048 & 3.146 & 3.164 & 5.017 & 5.031 \\
\hline 40.004 & 3.224 & 3.242 & 5.079 & 5.093 \\
\hline 29.995 & 3.307 & 3.316 & 5.144 & 5.151 \\
\hline 19.997 & 3.371 & 3.360 & 5.193 & 5.185 \\
\hline 19.013 & 3.375 & 3.361 & 5.196 & 5.186 \\
\hline 18.002 & 3.379 & 3.362 & 5.199 & 5.186 \\
\hline 17.003 & 3.383 & 3.362 & 5.202 & 5.186 \\
\hline 16.002 & 3.381 & 3.360 & 5.201 & 5.185 \\
\hline 15.002 & 3.379 & 3.358 & 5.199 & 5.183 \\
\hline 14.049 & 3.376 & 3.355 & 5.197 & 5.181 \\
\hline 13.026 & 3.366 & 3.350 & 5.189 & 5.177 \\
\hline 12.005 & 3.353 & 3.344 & 5.179 & 5.172 \\
\hline 11.023 & 3.338 & 3.336 & 5.168 & 5.166 \\
\hline 10.003 & 3.320 & 3.326 & 5.153 & 5.158 \\
\hline 9.005 & 3.302 & 3.312 & 5.140 & 5.147 \\
\hline 8.004 & 3.278 & 3.292 & 5.121 & 5.132 \\
\hline 7.005 & 3.243 & 3.265 & 5.093 & 5.111 \\
\hline 6.006 & 3.194 & 3.224 & 5.055 & 5.079 \\
\hline 5.003 & 3.116 & 3.158 & 4.993 & 5.026 \\
\hline 4.001 & 3.012 & 3.045 & 4.909 & 4.936 \\
\hline 2.999 & 2.799 & 2.831 & 4.732 & 4.759 \\
\hline 1.993 & 2.453 & 2.380 & 4.430 & 4.364 \\
\hline
\end{tabular}


Table S2: Experimental and calculated $\chi_{\mathrm{M}} T\left[\mathrm{~cm}^{3} \mathrm{~K} \mathrm{~mol}^{-1}\right]$ and $\mu_{\text {eff }}\left[\mu_{\mathrm{B}}\right]$ values for 3 .

\begin{tabular}{|c|c|c|c|c|}
\hline$T[\mathrm{~K}]$ & $\chi_{M} T_{\exp }$ & $\chi_{M} T_{\text {calc }}$ & $\mu_{\text {eff exp }}$ & $\mu_{\text {eff calc }}$ \\
\hline 330.020 & 2.700 & 2.762 & 4.647 & 4.701 \\
\hline 319.183 & 2.706 & 2.749 & 4.653 & 4.690 \\
\hline 309.384 & 2.705 & 2.738 & 4.652 & 4.680 \\
\hline 301.029 & 2.701 & 2.728 & 4.648 & 4.672 \\
\hline 290.279 & 2.698 & 2.715 & 4.646 & 4.661 \\
\hline 281.146 & 2.694 & 2.705 & 4.643 & 4.652 \\
\hline 269.750 & 2.688 & 2.691 & 4.637 & 4.640 \\
\hline 260.521 & 2.683 & 2.680 & 4.633 & 4.631 \\
\hline 250.273 & 2.677 & 2.668 & 4.627 & 4.620 \\
\hline 240.385 & 2.670 & 2.657 & 4.621 & 4.610 \\
\hline 230.296 & 2.661 & 2.645 & 4.614 & 4.600 \\
\hline 220.134 & 2.655 & 2.633 & 4.609 & 4.589 \\
\hline 209.970 & 2.643 & 2.620 & 4.598 & 4.579 \\
\hline 199.647 & 2.633 & 2.608 & 4.590 & 4.568 \\
\hline 189.890 & 2.623 & 2.596 & 4.580 & 4.557 \\
\hline 179.637 & 2.612 & 2.584 & 4.571 & 4.547 \\
\hline 169.653 & 2.600 & 2.572 & 4.561 & 4.536 \\
\hline 159.600 & 2.588 & 2.559 & 4.551 & 4.525 \\
\hline 149.649 & 2.574 & 2.547 & 4.538 & 4.514 \\
\hline 139.696 & 2.563 & 2.534 & 4.528 & 4.503 \\
\hline 129.735 & 2.541 & 2.522 & 4.508 & 4.492 \\
\hline 119.789 & 2.524 & 2.509 & 4.493 & 4.480 \\
\hline 109.822 & 2.521 & 2.496 & 4.491 & 4.468 \\
\hline 99.870 & 2.498 & 2.482 & 4.470 & 4.456 \\
\hline 89.915 & 2.487 & 2.468 & 4.460 & 4.444 \\
\hline 79.975 & 2.470 & 2.454 & 4.445 & 4.431 \\
\hline 70.014 & 2.449 & 2.438 & 4.426 & 4.416 \\
\hline 60.043 & 2.426 & 2.421 & 4.406 & 4.401 \\
\hline 50.051 & 2.404 & 2.402 & 4.385 & 4.384 \\
\hline 40.006 & 2.376 & 2.379 & 4.360 & 4.362 \\
\hline 29.995 & 2.343 & 2.347 & 4.330 & 4.333 \\
\hline 19.992 & 2.291 & 2.296 & 4.281 & 4.286 \\
\hline 19.035 & 2.283 & 2.289 & 4.273 & 4.279 \\
\hline 18.003 & 2.275 & 2.280 & 4.266 & 4.271 \\
\hline 17.002 & 2.265 & 2.271 & 4.256 & 4.262 \\
\hline 16.002 & 2.254 & 2.261 & 4.246 & 4.253 \\
\hline 15.002 & 2.242 & 2.250 & 4.235 & 4.242 \\
\hline 14.052 & 2.227 & 2.238 & 4.221 & 4.231 \\
\hline 13.028 & 2.208 & 2.223 & 4.203 & 4.217 \\
\hline 12.007 & 2.185 & 2.205 & 4.181 & 4.200 \\
\hline 11.024 & 2.163 & 2.186 & 4.159 & 4.182 \\
\hline 10.003 & 2.134 & 2.162 & 4.132 & 4.159 \\
\hline 9.003 & 2.103 & 2.133 & 4.102 & 4.131 \\
\hline 8.003 & 2.067 & 2.097 & 4.067 & 4.096 \\
\hline 7.005 & 2.022 & 2.052 & 4.022 & 4.051 \\
\hline 6.010 & 1.965 & 1.992 & 3.965 & 3.992 \\
\hline 5.003 & 1.885 & 1.909 & 3.883 & 3.908 \\
\hline 3.999 & 1.782 & 1.787 & 3.776 & 3.781 \\
\hline 2.997 & 1.611 & 1.598 & 3.590 & 3.575 \\
\hline 1.982 & 1.343 & 1.270 & 3.277 & 3.188 \\
\hline
\end{tabular}




\section{Geometry Optimizations}

Table S3: Cartesian coordinates / $\AA$ of geometry optimized $\left[\mathrm{Ni}_{2} \mathrm{~L}^{\mathrm{SO}}\left(\mathrm{O}_{2} \mathrm{CPh}\right)\right]^{+}\left(\mathbf{2}, \mathrm{M}^{\mathrm{S}}=5\right.$, quintet state, gas phase, PBE0/ def2-TZVP).

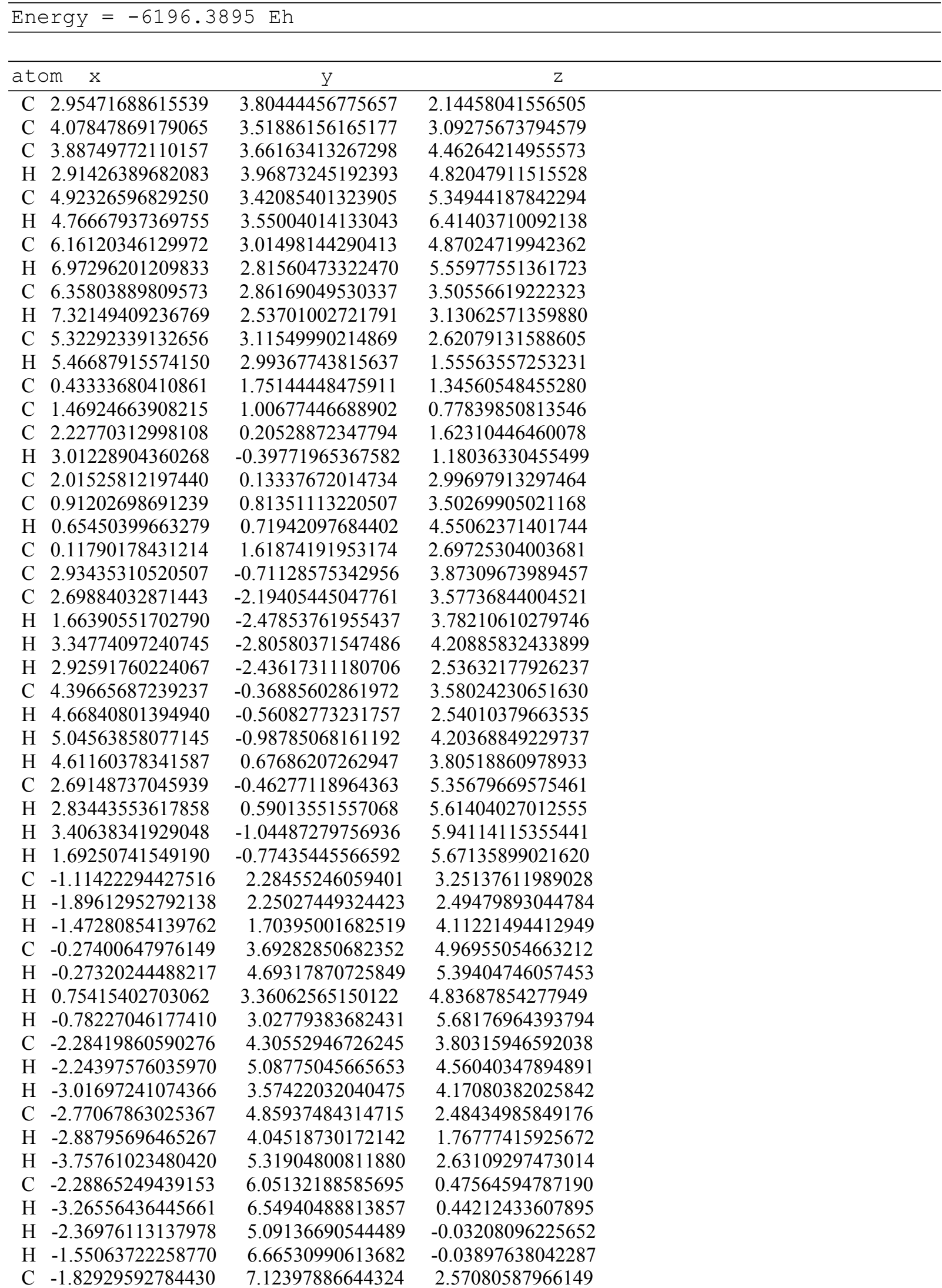


H $\quad-2.81713077549679$

H -1.63514385476258

C -0.78460594106762

H -0.77722383874150

H -1.03772379622176

C 1.44321596973314

H 1.52781243725389

H 2.42855024195706

H 1.06962844462821

C 1.08856637608761

H 0.39654424333002

H 1.12470246852358

C 2.44582879443109

C 3.57194044244298

H 3.43612407259417

C 4.84417023005839

C 4.92611838538689

H 5.88326568447007

C 3.82572408845822

C 2.57932308492537

C 6.10401016751697

C 5.79336070301004

H 6.72741847009599

H 5.21164374425180

H 5.24540043429614

C 6.93140962490808

H 7.82803309459325

H 7.25707505688976

H 6.35843184593840

C 6.92643097778070

H 7.83797746964828

H 6.35665837408108

H 7.21945369434839

C 3.95099648081500

H 4.80898030597501

H 3.05127272897964

C 5.39904862964056

H 5.61999792528968

H 5.33460403804378

H 6.22029613232068

C 4.21253009535880

H 4.62123582985809

H 4.92673032464111

C 2.87533755788151

H 2.59645615297945

H 2.98031481686437

C 0.51203391676401

H 0.57466846639833

H $\quad-0.28715387759021$

H 0.29032385685809

C 1.62882601079102

H 0.64428881507789

H 1.63866898991148

C 2.68084701120345

H 3.66279024487626

H 2.45350071134344

C 4.06616782007198

H 4.18918990267801

H 4.17954865676787

H 4.84961703128485

C 1.70868698632559
7.34746015299421

7.89738976621196

7.21538164108132

8.24475944641805

6.57190331626532

6.69025052978199

7.64147892033585

6.38586735644096

5.93322897826190

7.89887331294032

8.04915248888342

8.82949655587713

7.62619669039005

8.16437703063111

8.70458536892886

8.02630187888378

7.43811387024234

7.40399627166607

6.88719894272890

6.89209868941446

8.46482542874150

9.18998634650143

9.48125400830425

10.09954378705236

8.55186129817002

9.39722064054679

9.72233079051011

8.90466882469864

10.28445852444184

7.21167586908863

7.49074839124447

6.52038028606399

6.67842521188257

6.37809648418393

6.86842311888903

6.65818840400062

4.57639739954062

3.52091229149329

4.79531490591304

5.15799626480545

4.56154155597427

3.55440156672755

5.21802840157740

4.65812785605390

5.70797325882446

4.27692656488318

4.65736410310279

5.70051601348124

4.18291134038692

4.60172371809892

2.56351325806017

2.25164330190751

2.45212414086700

1.65926367964427

1.88399124078078

0.62342479475502

1.32005665879349

0.24300421769586

1.53087702995067

1.83867185682417

0.91316665297537
2.99398058137358

1.82753667710332

3.66633093909869

4.05590124948130

4.50627060422821

4.35277454810804

4.89611258900289

4.01096710645188

5.03318274528182

2.33988064865113

1.51080900498923

2.92455564247646

1.77902986402080

2.39231425115463

3.32147764194131

1.85794057065197

0.59859611088557

0.08921280310440

$-0.03974573618880$

0.59959316930955

2.58807042516435

3.89394324617440

4.37881471989270

3.72475172064689

4.59133755990090

1.70347568076748

2.23658760181945

0.78521220844445

1.42547572344526

2.91503358590071

3.44988476361716

3.54133978467448

2.00803851346815

$-1.44103558902680$

$-1.92279330648849$

$-1.98993315639807$

$-0.91628908203772$

$-1.04201496269270$

0.14539679102036

$-1.35621479066922$

$-2.98652264205371$

$-3.05384046526657$

$-3.50458302306875$

$-3.69105912102168$

$-3.78302929730885$

$-4.71506499770381$

$-3.29159087890840$

$-2.98369051166143$

$-2.72936032428743$

$-4.36487357113963$

$-3.35703718766624$

$-3.00821630366858$

$-4.45007108916650$

$-2.75927088606276$

$-3.17440080720981$

$-3.04904466166445$

$-0.83492391811082$

$-1.01296603996296$

0.22575978377730

$-1.38219337293056$

$-0.71024659677145$ 
H 1.99796477611023

H 0.78144694424587

N 2.75861816059804

N 1.77841551269359

N 4.13237159861432

$\mathrm{N} \quad 0.54952093244513$

$\mathrm{N}-1.85393645473896$

N -0.95353194308049

Ni 2.27872301018840

Ni 0.15018061866610

O 3.18465690272251

O 1.87749091856457

O 0.34188067047268

O $\quad-1.80294189907387$

S -0.40696702049130

S 1.22249284272201
$-0.12574458932461$

1.10935481799551

1.78329563828149

3.97983788203307

4.91594101329936

6.83249721849475

5.83231329538542

3.69409244889222

3.94531360817272

4.97054940394882

3.63415918290265

4.18954541646322

3.24819241430095

2.71356311905120

3.09702807082579

6.01385411132120
$-0.92405295878243$

$-1.23695699272390$

$-1.29567816141688$

$-2.98566426082249$

$-1.56473297652772$

3.20526702306121

1.86348282273592

3.68118252303926

$-0.86623165836125$

1.96583509943106

0.92630889800522

2.65999041916341

$-0.77523825892117$

0.26605732348825

0.51254177876359

$-0.08417322911595$

Figure S9. Molecular structure of the $\left[\mathrm{Ni}_{2} \mathrm{~L}^{\mathrm{SO} 2}\left(\mathrm{O}_{2} \mathrm{CPh}\right)\right]^{+}$cation (2) calculated at the PBE0/ def2-TZVP level of theory. Atom labels omitted. Bond lengths and angles are given in the Table below.

Table S4. Comparison of experimental (X-ray) and calculated (gas phase, PBE0/ def2-TZVP) bond lengths $/ \AA$ and angles $/{ }^{\circ}$ in $\left[\mathrm{Ni}_{2} \mathrm{~L}^{\mathrm{SO}}\left(\mathrm{O}_{2} \mathrm{CPh}\right)\right]^{+}(\mathbf{2})$.

\begin{tabular}{|l|l|l|l|l|l|l|}
\hline & $\exp ^{\mathrm{a})}$ & calc & & & $\exp ^{\mathrm{a})}$ & calc \\
\hline Ni1-N1 & $2.260(5)$ & 2.256 & & Ni2-N4 & $2.218(5)$ & 2.272 \\
\hline Ni1-N2 & $2.146(5)$ & 2.178 & & Ni2-N5 & $2.136(5)$ & 2.184 \\
\hline Ni1-N3 & $2.152(5)$ & 2.206 & & Ni2-N6 & $2.366(5)$ & 2.406 \\
\hline Ni1-O1 & $2.020(4)$ & 2.032 & & Ni2-O2 & $2.014(4)$ & 2.019 \\
\hline Ni1-O3 & $2.056(4)$ & 2.060 & & Ni2-S1 & $2.437(2)$ & 2.436 \\
\hline Ni1-S2 & $2.460(2)$ & 2.451 & & Ni2-S2 & $2.512(2)$ & 2.538 \\
\hline S1-O3 & $1.508(5)$ & 1.497 & & & & \\
\hline S1-O4 & $1.496(4)$ & 1.469 & & & & \\
\hline & & & & & & \\
\hline N1-Ni1-N2 & $82.1(2)$ & 83.0 & & N4-Ni2-N5 & $83.0(2)$ & 82.2 \\
\hline N1-Ni1-N3 & $103.1(2)$ & 100.5 & & N4-Ni2-N6 & $100.9(2)$ & 97.3 \\
\hline N1-Ni1-S2 & $162.1(2)$ & 164.0 & & N4-Ni2-S1 & $172.9(1)$ & 174.9 \\
\hline N1-Ni1-O1 & $85.6(2)$ & 85.8 & & N4-Ni2-O2 & $89.0(2)$ & 88.8 \\
\hline
\end{tabular}




\begin{tabular}{|l|l|l|l|l|l|l|}
\hline N1-Ni1-O3 & $83.4(2)$ & 83.3 & & N4-Ni2-S2 & $90.5(1)$ & 91.7 \\
\hline N2-Ni1-N3 & $82.2(2)$ & 83.0 & & N5-Ni2-N6 & $80.3(2)$ & 79.7 \\
\hline N2-Ni1-S2 & $102.7(1)$ & 101.4 & & N5-Ni2-S1 & $93.9(1)$ & 93.8 \\
\hline N2-Ni1-O1 & $163.5(2)$ & 164.4 & & N5-Ni2-O2 & $161.9(2)$ & 162.5 \\
\hline N2-Ni1-O3 & $84.7(2)$ & 80.4 & & N5-Ni2-S2 & $102.1(2)$ & 100.8 \\
\hline N3-Ni1-S2 & $94.7(1)$ & 95.3 & & N6-Ni2-S1 & $84.8(1)$ & 85.0 \\
\hline N3-Ni1-O1 & $90.0(2)$ & 88.4 & & N6-Ni2-O2 & $85.4(2)$ & 86.7 \\
\hline N3-Ni1-O3 & $164.4(2)$ & 162.3 & & N6-Ni2-S2 & $168.6(1)$ & 171.0 \\
\hline S2-Ni1-O1 & $92.4(1)$ & 92.3 & & S1-Ni2-O2 & $95.8(1)$ & 95.9 \\
\hline S2-Ni1-O3 & $80.0(1)$ & 82.3 & & S1-Ni2-S2 & $83.86(6)$ & 86.0 \\
\hline O1-Ni1-O3 & $104.8(2)$ & 109.2 & & O2-Ni2-S2 & $94.1(1)$ & 94.3 \\
\hline
\end{tabular}

Table S5: Cartesian coordinates / $\AA$ of geometry optimized $\left[\mathrm{Ni}_{2} \mathrm{~L}^{\mathrm{SO} 4}\left(\mathrm{O}_{2} \mathrm{CPh}\right)\right]^{+}(\mathbf{3}$, gas phase, PBE0/ def2-TZVP).

\begin{tabular}{|c|c|c|c|}
\hline Ener & $=$ & & \\
\hline atom & $x$ & $\mathrm{y}$ & $\mathrm{z}$ \\
\hline
\end{tabular}

C 4.31269121433722

H 3.28305024611688

H 4.77445374145690

H 4.85682669935100

C 5.85081524987346

H 5.96947540111454

H 6.41764732443152

H 6.29597061892039

C 3.80679172320442

H 2.74493920449280

H 4.33890474899533

H 3.92969792214655

C 2.22786000106538

C 2.35066219490842

C 3.04199778699202

H 3.16240314746829

C 3.60891753809282

C 3.50535982971259

H 3.98585765798904

C 2.83228040772352

C 2.87799078352462

H 3.14687614169716

H 3.67353054545229

C 1.42223660835435

H 1.36242696457747

H 2.25612895490284

H 0.49703127842466

C 1.77414856642704

H 1.00026793974236

H 2.73691096215176

C 1.68517878134432

H 2.53931881667520

H 1.76289184722350

C 0.77533477395277

H 1.61687056045821

H -0.09443103972238

H 1.00858214175278
20.22021377505196 20.58380633798060 20.58233863222054 20.66994736762727 18.27566580976728 17.20803752853299 18.81593700030202 18.49690261485568 18.31042324215941 18.55916959224447 18.85087868165046 17.24331804669555 16.66631893881449 18.05059164650618 18.69088131110211 19.76531324747269 18.00948588402072 16.61997193856030 16.04045203787581 15.94004477014427 14.43431925484989 14.11464192917534 14.10127603653065 14.04142005704732 15.11592967701347 13.64013252747099 13.59858726106739 12.30916441196906 11.82223404865201 11.96243657360823 11.90186082050766 12.31375995475325 10.80934128685216 12.50099336976641 13.17122852482598 12.91399521382476 11.51278432541936
21.43439524922246 21.38926990808533 22.35599164982971 20.60247882461707 21.36822550809346 21.16840743790714 20.60667349918740 22.34081060850280 19.95397573964206 19.87931549504553 19.16786766999403 19.75557400014750 24.48130686625931 24.54211093478594 23.51019282117553 23.57683093741973 22.43861858968210 22.44941580512210 21.66875770345723 23.44987277507654 23.47877477628850 24.48419884338667 22.79885489810322 21.66019756286586 21.50726873490428 21.06915867556129 21.30852589271289 23.27326754955307 22.68194602313057 22.86934057656732 24.73114576856468 25.26966686620896 24.80770231954866 26.85889960549368 27.01062078159585 27.36629255475090 27.27491181372586 
C $\quad-0.68066049556175$

H -1.38715315605225

H $\quad-0.38444591869989$

C -1.36346975433281

H -0.71195156915183

H $\quad-2.23982506365772$

C -1.94638793551384

H -2.12994862051993

H -1.05539215879917

H $\quad-2.78879961658664$

C -3.05511676531451

H -2.95471012639564

H -3.78632149978363

C -3.63259106687812

C -3.44691222575148

C -4.18929441227758

C -5.06065840860834

H -5.63794644102841

C -5.23332259993992

C -4.52736370034766

H -4.68724915195789

C -4.14374267875547

H -4.19262206982699

H $\quad-5.03647520718200$

C -3.06412184994570

H -3.06664287081446

H -3.99188232517438

H -2.22257970181731

C -2.95938144718817

H -2.31397386703825

H -3.96362891665206

C -2.50632669675814

H -2.49038858830130

H $\quad-3.24098401610633$

C -1.17829811407549

H $\quad-0.24895818203680$

H -1.23862988405493

H $\quad-2.01256604332257$

C -0.03778829350129

H $\quad-0.19172691078179$

H 0.79194735748008

C 0.33128697404258

H 1.22880958880781

H $\quad-0.45220388514749$

C 0.43074517422315

H -0.51457354240634

H 1.23700529479928

H 0.47496708694719

C 1.92609420901587

H 2.05614104877988

H 2.61157124034326

C 4.37937465561181

C -6.17626617317855

C -7.58199215746363

H $\quad-8.26170564017785$

H -7.96908645002540

H -7.60489675333935

C -6.29639077720960

H -5.33731185145104

H -6.70622662469444

H $\quad-6.97576627130469$
11.49111443740899

11.81096919878302

10.46224021971971

11.52000944127596

11.11644960973734

10.85798309382474

12.93294117881503

13.95824543412914

12.56585858014570

12.30052268204489

13.19064062033751

13.02785636506872

12.46281331576193

14.56145782810686

15.65790264420200

16.82990894529421

16.91286872739667

17.82229790011812

15.85655977573345

14.69051723815633

13.82246611743927

17.94257868906063

17.50357838465895

18.56957446065067

19.57948919565461

18.89264589695870

20.16734961252893

20.25575336803955

19.75549412942716

20.59122157958476

20.17712383123385

19.10966829989892

19.86884007260809

18.36199668369299

17.37255738796267

16.81307614668750

17.83759419085302

16.68845817249302

19.27300834638667

19.88866363211065

18.59887075280998

20.16488679872819

20.73972708382166

20.89926189743820

20.31799129738560

20.85318491279698

21.06505754059985

19.74864297573108

18.86750283572388

18.27540823327503

19.72541527397194

18.70335286895041

15.98839056726717

16.36645586250071

16.41022215928878

15.62406864524920

17.33982510783443

14.68501204483608

14.35657214505893

13.88190264784526

14.83258937903570
25.30495331923786

26.07111114087862

25.55061920966578

23.96078228916799

23.18710496277686

23.99309179694788

22.12796482940497

21.81909571376822

21.62386186682513

21.81557250401531

24.22089860922599

25.28941408207012

23.84063430869506

23.96285655321132

24.79663312242308

24.61607159303694

23.54085581278443

23.41293791123655

22.64716671563710

22.90364107671866 22.27821015446306 25.63633436975654 26.63227838643023 25.50815089959697 24.31795496893253 23.47604247603200 24.30442743042973 24.20938578467813 26.70048912749582 26.43403459676463 26.85286941819428 27.99416628542406 28.78741512443171 28.29633108325505 28.94962699803549 28.86445567418378 29.94176588901383 28.81319768204505 28.13789958556331 29.03447081346141 28.35254732657519 26.97872063324526 27.24805855917141

26.79728954878696 24.59947004633837

24.64325108673710 24.60439056558937 23.67551487672873 25.74304878512847 26.64312678568723 25.80663570827318 21.31958084711286 21.45915194023816 21.93752410606728 21.08334641421993 22.63838070170540 22.43166202781885 20.67450433556985 20.26548783405577 21.29213284298438 19.83327411702547 


$\begin{array}{cccc}\mathrm{C} & -5.63247405962227 & 17.08114262625010 & 20.53225525143262 \\ \mathrm{H} & -4.62375219791677 & 16.84166057065140 & 20.18568285646653 \\ \mathrm{H} & -6.27770267916227 & 17.19043296585290 & 19.65744650091936 \\ \mathrm{H} & -5.59143048924119 & 18.04725560539005 & 21.03945016910241 \\ \mathrm{C} & -0.84445963805634 & 16.79611385946678 & 22.95198144031593 \\ \mathrm{C} & -1.01020849542263 & 17.35694677887825 & 21.57414316126325 \\ \mathrm{C} & -1.07588175343021 & 16.52280879970377 & 20.46226664567496 \\ \mathrm{H} & -1.00549579894175 & 15.45259106699336 & 20.60329319108282 \\ \mathrm{C} & -1.22830835827413 & 17.05738529339061 & 19.19298503867618 \\ \mathrm{H} & -1.27493577153495 & 16.40207470720517 & 18.33141747848817 \\ \mathrm{C} & -1.32130970587613 & 18.43207407420192 & 19.02364840139805 \\ \mathrm{H} & -1.44412847500075 & 18.85047530688020 & 18.03168037592116 \\ \mathrm{C} & -1.25796068130420 & 19.27045285828538 & 20.12782327957588 \\ \mathrm{H} & -1.33303195449882 & 20.34412837536034 & 20.00090675987186 \\ \mathrm{C} & -1.09872845256671 & 18.73329268805531 & 21.39333691962729 \\ \mathrm{H} & -1.04273703893404 & 19.37406053425140 & 22.26080282912137 \\ \mathrm{O} & -0.68991057424629 & 15.55758384380714 & 23.04802194440008 \\ \mathrm{O} & -0.87193808066814 & 17.61461337531063 & 23.89918573322782 \\ \mathrm{O} & -1.40200929896037 & 14.51406138147253 & 25.95052687007093 \\ \mathrm{O} & -3.14043671653950 & 15.51340245158629 & 27.36191240125110 \\ \mathrm{O} & 0.56035430999669 & 16.61809434230838 & 26.51614172570455 \\ \mathrm{O} & 2.42523265511911 & 15.03150339568091 & 26.46982348919271 \\ \mathrm{~S} & 1.35901872960709 & 15.66112056493064 & 25.68067073635208 \\ \mathrm{~S} & -2.30382757736415 & 15.69203422554310 & 26.16888524303003 \\ \mathrm{~N} & -0.08774316855804 & 14.21012430605686 & 24.42243365192215 \\ \mathrm{Ni} & -1.03816510541757 & 17.72260946819046 & 25.89228592061236 \\ \mathrm{~N} & 1.62467155497721 & 13.75550147295143 & 23.07933526581364 \\ \mathrm{~N} & 0.47842153634363 & 12.38914367898152 & 25.42051619332394 \\ \mathrm{~N} & -1.75643588444914 & 12.88088247945935 & 23.57266043914412 \\ \mathrm{~N} & -2.95495835770331 & 18.82503981913868 & 25.56413097531609 \\ \mathrm{~N} & -1.21021717359141 & 18.41253685525453 & 27.90623921417610 \\ \mathrm{~N} & 0.54615726274553 & 19.41280208744474 & 25.73635137632460\end{array}$

a) There are two crystallographically independent molecules A and B in the unit cell. The values calculated here utilized the fractional coordinates of the non disordered cation A.

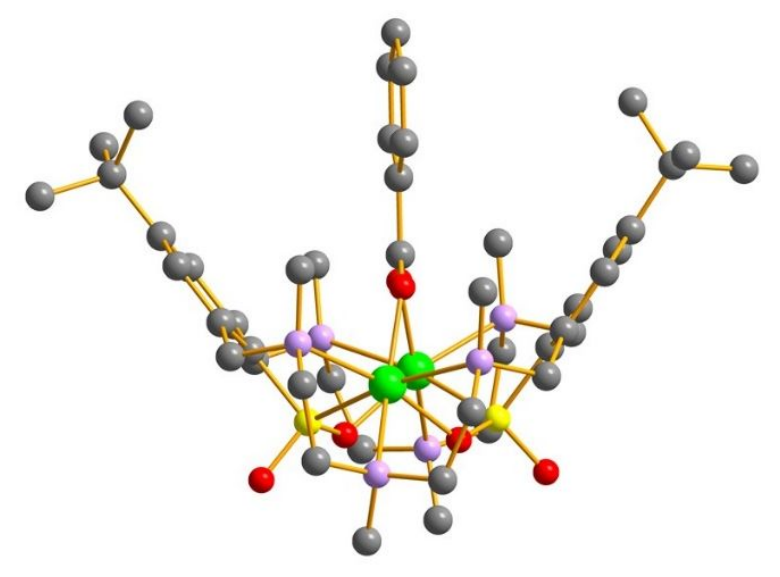

Figure S10. Molecular structure of the $\left[\mathrm{Ni}_{2} \mathrm{~L}^{\mathrm{SO}}\left(\mathrm{O}_{2} \mathrm{CPh}\right)\right]^{+}$cation (3) calculated at the PBE0/ def2-TZVP level of theory. Atom labels omitted. Bond lengths and angles are given in the Table below. 
Table S6. Comparison of experimental (X-ray) and calculated (PBE0/ def2-TZVP) bond lengths $/ \AA$ and angles $/{ }^{\circ}$ in $\left[\mathrm{Ni}_{2} \mathrm{~L}^{\mathrm{SO} 4}\left(\mathrm{O}_{2} \mathrm{CPh}\right)\right]^{+}(3){ }^{\text {a) }}$

\begin{tabular}{|l|l|l|l|l|l|l|}
\hline & $\exp ^{\text {a) }}$ & calc & & & exp $^{\text {a) }}$ & calc \\
\hline Ni1-N1 & $2.143(3)$ & 2.223 & & Ni2-N4 & $2.161(3)$ & 2.235 \\
\hline Ni1-N2 & $2.105(3)$ & 2.152 & & Ni2-N5 & $2.101(3)$ & 2.136 \\
\hline Ni1-N3 & $2.240(3)$ & 2.296 & & Ni2-N6 & $2.227(3)$ & 2.322 \\
\hline Ni1-O1 & $2.005(3)$ & 2.017 & & Ni2-O2 & $1.999(3)$ & 2.041 \\
\hline Ni1-O3 & $2.047(2)$ & 2.038 & & Ni2-O5 & $2.013(2)$ & 2.003 \\
\hline Ni1-S1 & $2.533(1)$ & 2.405 & & Ni2-S2 & $2.530(1)$ & 2.500 \\
\hline S1-O5 & $1.496(3)$ & 1.501 & & S2-O3 & $1.492(3)$ & 1.500 \\
\hline S1-O6 & $1.483(3)$ & 1.468 & & S2-O4 & $1.494(3)$ & 1.468 \\
\hline & & & & & & \\
\hline N1-Ni1-N2 & $85.66(11)$ & 84.5 & & N4-Ni2-N5 & $85.35(12)$ & 84.8 \\
\hline N1-Ni1-N3 & $103.49(11)$ & 102.6 & & N4-Ni2-N6 & $103.65(12)$ & 102.5 \\
\hline N2-Ni1-N3 & $83.98(12)$ & 82.7 & & N5-Ni2-N6 & $84.94(12)$ & 83.3 \\
\hline N1-Ni1-S1 & $89.41(8)$ & 88.6 & & N4-Ni2-S2 & $89.65(9)$ & 89.0 \\
\hline N2-Ni1-S1 & $97.59(9)$ & 96.3 & & N5-Ni2-S2 & $96.19(9)$ & 97.0 \\
\hline N3-Ni1-S1 & $167.09(8)$ & 168.6 & & N6-Ni2-S2 & $166.71(9)$ & 168.5 \\
\hline O1-Ni1-N1 & $89.08(10)$ & 87.4 & & O2-Ni2-N4 & $88.08(11)$ & 87.2 \\
\hline O1-Ni1-N2 & $167.95(12)$ & 163.7 & & O2-Ni2-N5 & $168.07(11)$ & 164.2 \\
\hline O1-Ni1-N3 & $86.72(11)$ & 85.3 & & O2-Ni2-N6 & $86.95(11)$ & 85.2 \\
\hline O1-Ni1-O3 & $99.54(10)$ & 102.6 & & O2-Ni2-O5 & $101.27(10)$ & 102.1 \\
\hline O1-Ni1-S1 & $93.18(8)$ & 97.6 & & O2-Ni2-S2 & $93.71(8)$ & 96.5 \\
\hline O3-Ni1-N1 & $167.57(11)$ & 168.5 & & O5-Ni2-N4 & $167.14(11)$ & 169.6 \\
\hline O3-Ni1-N2 & $87.47(11)$ & 87.0 & & O5-Ni2-N5 & $86.86(11)$ & 87.1 \\
\hline O3-Ni1-N3 & $86.05(11)$ & 84.0 & & O5-Ni2-N6 & $85.81(11)$ & 83.1 \\
\hline O3-Ni1-S1 & $81.23(8)$ & 84.6 & & O5-Ni2-S2 & $81.03(8)$ & 85.4 \\
\hline There & & & & \\
\hline
\end{tabular}

a) There are two crystallographically independent molecules A and B in the unit cell. The values given here refer to the non-disordered cation $\mathrm{A}$. 


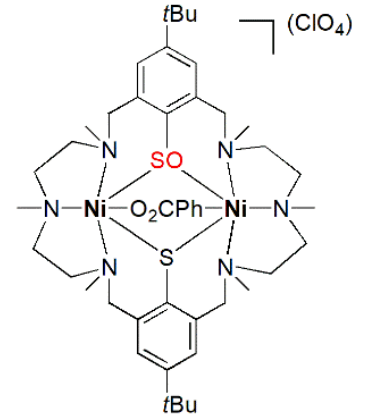

$\left[\mathrm{Ni}_{2} \mathrm{~L}^{\mathrm{SO}}\left(\mathrm{O}_{2} \mathrm{CPh}\right)\right]^{+}$

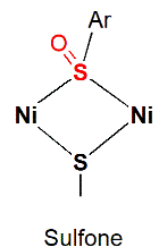

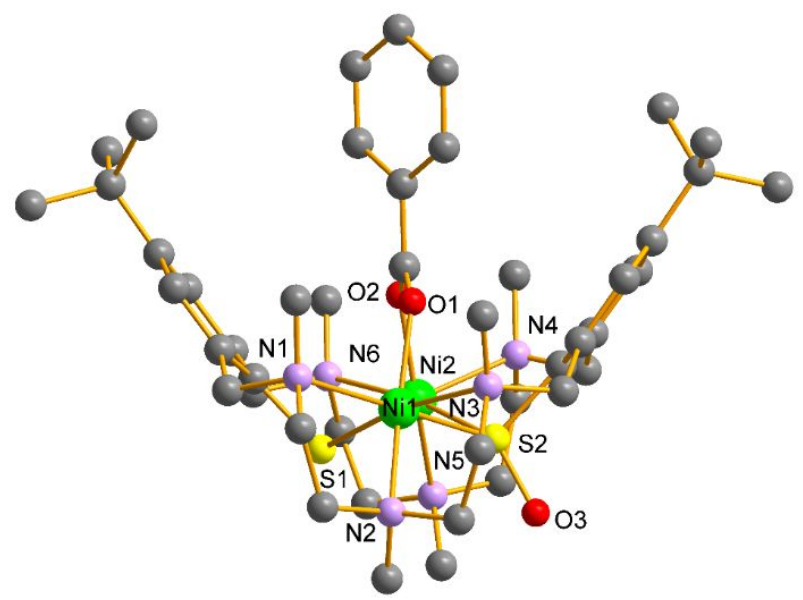

Figure S11. Molecular structure of the $\left[\mathrm{Ni}_{2} \mathrm{~L}^{\mathrm{SO} 2}\left(\mathrm{O}_{2} \mathrm{CPh}\right)\right]^{+}$cation (2, sulfone form) calculated at the PBE0/ def2-TZVP level of theory. Bond lengths and angles are given in Table S8 below.

Table S7: Cartesian coordinates / $\AA$ of geometry optimized $\left[\mathrm{Ni}_{2} \mathrm{~L}^{\mathrm{SO}}\left(\mathrm{O}_{2} \mathrm{CPh}\right)\right]^{+}$(pre-2, sulfoneform, PBE0/ def2-TZVP).

\begin{tabular}{lccc}
\hline Energy $=-6121.1909$ Eh & \\
\hline \multicolumn{4}{c}{ y } \\
\hline atom $\mathrm{y}$ & & \\
\hline & & \\
$\mathrm{Ni}$ & -0.13871527676357 & 2.14383194388526 & 11.85860214766554 \\
$\mathrm{Ni}$ & 0.74266825757553 & 5.48051839679778 & 11.54417790509799 \\
$\mathrm{~S}$ & 1.83476301636102 & 3.54902700577435 & 12.37452948666707 \\
$\mathrm{~S}$ & -1.23521575940995 & 4.26001498396490 & 12.44855982399866 \\
$\mathrm{O}$ & -0.17084384555243 & 2.61188389229184 & 9.89287775321092 \\
$\mathrm{O}$ & 0.79307382939580 & 4.62735490933246 & 9.72784925140673 \\
$\mathrm{~N}$ & 1.18595086070191 & 0.42343131668530 & 11.30505083822312 \\
$\mathrm{~N}$ & -0.30413543157649 & 1.11031844134534 & 13.74786749978987 \\
$\mathrm{~N}$ & -2.19929783668889 & 1.08537347039505 & 11.57540704823084 \\
$\mathrm{~N}$ & -0.57440737892678 & 7.07261806639166 & 10.74037548011381 \\
$\mathrm{~N}$ & 0.95583018805775 & 6.78370213266676 & 13.23788863074956 \\
$\mathrm{~N}$ & 2.82643783560181 & 6.46894073379777 & 11.01075028790237 \\
$\mathrm{C}$ & 3.02560128295982 & 3.14803531277672 & 11.12171312678434 \\
$\mathrm{C}$ & 3.18404522412153 & 1.82938175969185 & 10.71421727341790 \\
$\mathrm{C}$ & 4.00143840635508 & 1.55713779956363 & 9.62987073849155
\end{tabular}


H 4.12205281646105

C 4.67849399606824

C 4.58812232873871

H 5.17432797515295

C 3.78817931518849

C 2.60855850973978

H 3.18640856293238

H 2.74450839645685

C 1.05864823541734

H 1.35583830166719

H 0.02945817946503

H 1.69206370230312

C 0.75927134886941

H 1.48957396688466

H -0.16291283193451

C 0.57711888232450

H 0.20867643860996

H 1.54696272167014

C 0.08911067997999

H $\quad-0.53440809770641$

H 1.12832620739381

H -0.05659501059538

C -1.71005723105261

H -2.23906990071430

H $\quad-1.79865443993984$

C -2.35003994940515

H -1.90673094678233

H -3.41164935828042

C -2.39494328879940

H -2.12899759734502

H -3.43788324408594

H $\quad-1.76699216973889$

C -3.25920709790440

H -3.23220420214014

H -4.22728439072744

C -3.17687270134645

C -2.39761970081129

C -2.57724111005229

C -3.38332503903834

H $\quad-3.49961376004818$

C -4.05436659213781

C -3.96907091026051

H -4.55115222901474

C -2.00950151909562

H -2.56881770591202

H -2.16352034056040

C -0.43683421504876

$\mathrm{H} \quad-0.76830016746974$

H $\quad 0.59820237471114$

H $\quad-1.04163620694697$

C -0.14031909007505

H -0.88207993394657

H 0.76767020013376

C 0.08149901689757

H 0.47159582787288

H $\quad-0.87977887705441$

C 0.59326364383102

H $\quad-0.45380532596581$

H $\quad 0.75833538817410$

H 1.21347197158161

C 2.37867833789324
0.52435748047821 2.56254529719570 3.85556096653510 4.65193110213570 4.15600682112782 0.72163130020124 $-0.19397990286226$ 0.98146399114748 $-0.06433435867453$ 0.71754502099691 $-0.32559249631099$ $-0.94846082860682$ $-0.60840291191813$ $-1.43017273886428$ $-1.03769177683553$ $-0.06801774827152$ $-0.87318898370464$ 0.22764063976808 1.93364509839041 2.82771069003931 2.24732326028818

1.38008884899174 0.73201007746977 1.63485125714827 0.01566019600560 0.16102921275201 $-0.79835703363912$ $-0.03996849334257$ 0.39439737674275 1.06406232059239 0.06964091328592 $-0.49256124612531$ 2.10316621793085 2.48238768806011 1.60174544493580 3.27303712289822 4.37320465967429 5.57119545018761 5.59071678566727 6.53282175788044 4.45772824173237 3.32351686585781 2.44607885122128 6.83766358423620 7.68964923800780 6.82965158455190 7.32027727509347 6.44325090263156 7.50480205557619 8.18639779832474 8.24634423260119 9.05505558732149 8.62221996382023 7.93960496062847 8.83660473853088

7.72214837828032 6.17867517156828 5.88175712558663 6.89251050619400 5.29583564129905 7.14348715452085
9.32136713904925 8.94309384665346 9.45084600958155 9.00789037165893 10.54658120527872 11.54137919668889 11.35038309931176 12.59141577726207 9.93139113529478 9.23799977862109 9.71382127245380 9.77583483841611 12.25094362151317 12.28754567923901

11.86716703857672 13.65804501322353 14.30665321529359 14.05872814868033 14.89918279205835 14.92169968284914 14.80067343471466 15.83565897266106 13.94310314876544 14.25060459291410 14.77101316456760 12.70242019077349 12.44329787551152 12.90550130837407 10.30455495707587 9.48970727754586 10.18586003740901 10.25695470604823 11.70676262075033 12.72861169612583 11.56403532793813 10.78821582235247 11.14199704962915 10.44381831756042 9.31484853985042 8.78895465250059 8.85732918940903 9.65032183117299 9.39869729335000 11.00015476520426 10.58731395693692 12.08048405209914 9.30536611150750 8.75540633557892 9.04462370353595 9.00428489070334 11.50265366128682 11.42783959370420 11.03591723597459 12.97388609161915 13.47232751434970

13.43978143861442 14.52895482126275 14.51331726343171 15.34617663443212 14.69000011908551 13.32969781813715 
H 2.88865009047827

H 2.51562369964078

C 2.99499869688272

H 2.54898938124450

H 4.05963817567058

C 2.98986967440337

H 2.68483123192747

H 4.03319059587662

H 2.37607761024053

C 3.89128704247013

H 3.92520123314813

H 4.85163706658804

C 5.52180561158355

C 4.63434094664248

H 5.23012322479096

H 3.82989142615234

H 4.17623257217683

C 6.13028471146836

H 5.35792113374141

H 6.69728137452125

H 6.81886333183572

C 6.65335496788151

H 6.26801820763369

H 7.30871443410205

H 7.25432132096338

C -4.89130002699156

C -6.08718955973190

H $\quad-5.76560254432024$

H -6.73187248688995

H -6.68248534898700

C -4.03234852440515

$\mathrm{H} \quad-3.63732797003041$

H -4.62590661197918

H $\quad-3.18238297931006$

C -5.41012810577742

H $\quad-5.96012063136779$

H -6.09543238436438

H $\quad-4.58921171647211$

C 0.30118379832513

C 0.29936232226463

C 1.34348023555806

H 2.15051794508957

C 1.35859750367776

H 2.18233159221548

C 0.31575176668897

H 0.32254867618487

C -0.73663122038601

H -1.55609802900830

C -0.73716960118194

H -1.55216408612960

O 2.54455470856986
6.26928665221987 7.96413471009014 7.53021716511005 8.44713034393255 7.75382138888318 6.99396561924022 6.23581011300991 7.28139762479615 7.88144244344593 5.47694023602809 5.26804433357498 5.93852512619243 2.19967447910994 1.49090795762612 1.19857722494974 2.14749455926870 0.58798970491698 3.42446336906298 4.12071980012503 3.10891557839021 3.95913571639991 1.26521148002330 0.35802484385592 1.76028981900994 0.96518423639334 4.50664630177510 5.43446241041165 6.43275414921233 5.03906221209526 5.53055601822819 5.02972154252919 6.02831840620143 5.07935894598748 4.36726651399936 3.12763781884186 3.20079754336018 2.71291069612517 2.42144512009024 3.57802837700223 3.46413005480645 4.01279889285596 4.50720531117018 3.89840909235259 4.31139910161412 3.25487232348758 3.16911302514755 2.72011440844697 2.22907733725458 2.81146577036561 2.40202300285448 3.56883259581106
13.74127997112805

14.04742193941225

12.00878991317098

11.62706131560760

12.16841622658234

9.65885967238269

8.93973410761574

9.46756921501669

9.52019502691485

11.24795715943725

12.31775348103401

10.97666260098805

7.73043360201149

6.70324920513333

5.83536148608906

6.36396780363546

7.11173861370744

7.05644003643200

6.72018988368771

6.17881254809150

7.71475580361874

8.17327626969153

8.64293440465257

8.89306599501643

7.31153084179106

7.58856044212489

7.82262711009804

8.12855247953211

8.61090170013000

6.91145345062099

6.43443791490815

6.63318764687702

5.51853814516136

6.25542284411535

7.19059423785792

6.25027094976071

7.93291458347090

7.04086596953888

9.24871974610095

7.75988066726994

7.02326516475778

7.54872623243557

5.64266168078911

5.07198754793251

4.98955200851763

3.90931610783105

5.72019598616391

5.20860914027698

7.10311023807883

7.68645443522173

13.72418227965784 
Table S8. Calculated bond lengths $/ \AA$ and angles $/{ }^{\circ}$ in $\left[\mathrm{Ni}_{2} \mathrm{~L}^{\mathrm{SO}}\left(\mathrm{O}_{2} \mathrm{CPh}\right)\right]^{+}$(pre-2, sulfone), ${ }^{\mathrm{a})}$ PBE0/ def2-TZVP

\begin{tabular}{|l|l|l|l|l|}
\hline & sulfone & & & sulfone \\
\hline Ni1-N1 & 2.241 & & Ni2-N4 & 2.217 \\
\hline Ni1-N2 & 2.160 & & Ni2-N5 & 2.148 \\
\hline Ni1-N3 & 2.334 & & Ni2-N6 & 2.367 \\
\hline Ni1-O1 & 2.021 & & Ni2-O2 & 2.007 \\
\hline Ni1-S1 & 2.477 & & Ni2-S1 & 2.369 \\
\hline Ni1-S2 & 2.455 & & Ni2-S2 & 2.491 \\
\hline S1-O3 & 1.525 & & Ni1-Ni2 & $\mathbf{3 . 4 6 5}$ \\
\hline & & & & \\
\hline & & & & \\
\hline N1-Ni1-N2 & 83.9 & & N4-Ni2-N5 & 84.8 \\
\hline N1-Ni1-N3 & 98.3 & & N4-Ni2-N6 & 98.1 \\
\hline N1-Ni1-S2 & 170.1 & & N4-Ni2-S1 & 170.5 \\
\hline N1-Ni1-O1 & 87.0 & & N4-Ni2-O2 & 89.5 \\
\hline N1-Ni1-S1 & 90.9 & & N4-Ni2-S2 & 90.7 \\
\hline N2-Ni1-N3 & 79.7 & & N5-Ni2-N6 & 80.6 \\
\hline N2-Ni1-S2 & 99.7 & & N5-Ni2-S1 & 99.9 \\
\hline N2-Ni1-O1 & 163.8 & & N5-Ni2-O2 & 165.7 \\
\hline N2-Ni1-S1 & 98.6 & & N5-Ni2-S2 & 95.1 \\
\hline N3-Ni1-S2 & 91.5 & & N6-Ni2-S1 & 90.8 \\
\hline N3-Ni1-O1 & 88.5 & & N6-Ni2-O2 & 87.2 \\
\hline N3-Ni1-S1 & 170.4 & & N6-Ni2-S2 & 169.8 \\
\hline S2-Ni1-O1 & 91.5 & & S1-Ni2-O2 & 87.7 \\
\hline S2-Ni1-S1 & 79.4 & & S1-Ni2-S2 & 80.8 \\
\hline O1-Ni1-S1 & 94.8 & & O2-Ni2-S2 & 98.1 \\
\hline
\end{tabular}

a) This structure is less stable than the sulfenate form. 


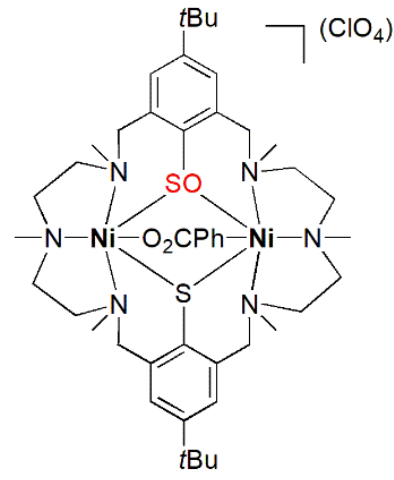<smiles>N1OSN[AlH2]1</smiles>

Sulfenate

$\left[\mathrm{Ni}_{2} \mathrm{~L}^{\mathrm{SO}}\left(\mathrm{O}_{2} \mathrm{CPh}\right)\right]^{+}$

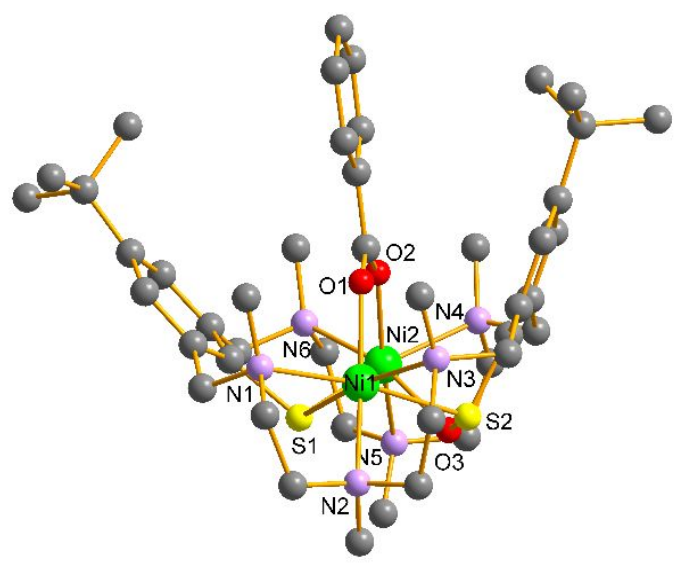

Figure S12. Molecular structure of the $\left[\mathrm{Ni}_{2} \mathrm{~L}^{\mathrm{SO}}\left(\mathrm{O}_{2} \mathrm{CPh}\right)\right]^{+}$cation (pre-2, sulfenate form) calculated at the PBE0/ def2-TZVP level of theory. Bond lengths and angles are given in Table S10 below

Table S9: Cartesian coordinates / $\AA$ of geometry optimized $\left[\mathrm{Ni}_{2} \mathrm{~L}^{\mathrm{SO}}\left(\mathrm{O}_{2} \mathrm{CPh}\right)\right]^{+}$(sulfenate form, PBE0/ def2-TZVP level of theory).

\begin{tabular}{lccc}
\hline Energy $=-6121.2041$ Eh & \\
\hline \multicolumn{4}{c}{ Y } \\
\hline atom x & \multicolumn{3}{l}{ z } \\
\hline C & 2.94612478096183 & 3.82378490959090 & 2.17909181648594 \\
C & 4.10342492900299 & 3.52688027730320 & 3.08300198611198 \\
C & 3.99521864049588 & 3.73762070451974 & 4.45338218584287 \\
H & 3.05833611621787 & 4.10879390123550 & 4.84729322614936 \\
C & 5.06303296583872 & 3.47614028431795 & 5.29529619457912 \\
H & 4.97170355461142 & 3.65837913315922 & 6.35974692129240 \\
C & 6.24911482977391 & 2.98022511841567 & 4.77148110319516 \\
H & 7.08419002155538 & 2.76121240065413 & 5.42643432438415 \\
C & 6.36348423333533 & 2.76186957676347 & 3.40613328398418 \\
H & 7.28600811874032 & 2.36768657348118 & 2.99625967726549 \\
C & 5.29821701562237 & 3.03838138554829 & 2.56602453564561 \\
H & 5.37874740756928 & 2.86751689501986 & 1.50140020942751 \\
C & 0.48184021941343 & 1.81542060413015 & 1.39152501079610 \\
C & 1.48321037307587 & 1.03533697498613 & 0.80407072410855 \\
C & 2.22603566762634 & 0.20658593771232 & 1.63738712944799 \\
H & 2.98963463520679 & -0.41143799364848 & 1.17766634050934 \\
C & 2.02241280121238 & 0.11258767849637 & 3.01006308142313 \\
C & 0.93222228858345 & 0.80597576078166 & 3.52817974306825
\end{tabular}


H $\quad 0.66587693725400$

C 0.15741542733822

C 2.92737963081802

C 2.68832843576782

H 1.65067985975092

H 3.33055908252842

H 2.92044070270445

C 4.39338407918236

H 4.66856294729098

H 5.03757033187197

H 4.61010184673298

C 2.67367117131860

H 2.82028872897212

H 3.37920915476461

H 1.66909635725816

C -1.07762432178421

H -1.84562847432622

H -1.46906218268834

C -0.26704695732529

H $\quad-0.28694137525346$

H 0.76806822922513

H -0.77201071758028

C -2.26872411571826

H -2.24635295134011

H $\quad-2.99871555529350$

C -2.74701539345732

H -2.84933880735474

H $\quad-3.74538499811867$

C -2.24624354863636

H -3.24006400163176

H $\quad-2.27477662372575$

H -1.51578137162002

C -1.84467335389482

H -2.84217627220055

H -1.64940028677355

C -0.81824805261590

H -0.83272661991108

H $\quad-1.07636975767782$

C 1.40335838114296

H 1.46414777453475

H 2.39780668772335

H 1.03053927163131

C 1.06755293976877

H $\quad 0.37987263460720$

H 1.09998698821728

C 2.42662720297645

C 3.54959165649660

H 3.41014025199276

C 4.82360351908864

C 4.90939439395872

H 5.86849892815456

C 3.81136253275444

C 2.56357654988334

C 6.08089671832397

C 5.76620029583199

H 6.69860929985441

H 5.17874887274797

H 5.22222014892866

C 6.89581921262689

H 7.79316760686566

H 7.21859631916957
0.69544391740117 1.64106604764640 $-0.76843618768360$ $-2.24159324853326$ $-2.52647697301949$ $-2.87494402627295$ $-2.45512712887823$ $-0.42640953439247$ $-0.59188675819351$ $-1.06326307885027$ 0.61307324648109 $-0.56269474316340$ 0.48159840531661 $-1.16602759656675$ $-0.87586136256317$ 2.30670833981615 2.27348458233677

1.72823526821674 3.72166063628863 4.72143565977269 3.40748514244781

3.04664574497555 4.31537370146989

5.11130221320106 3.58314848871503 4.84512951021349 4.01704413234922 5.28658071250858 6.01118845419018 6.47179422967318 5.04763074902802 6.64159929063146 7.12060900974073 7.33692508829644 7.88557535322365 7.24408810241719 8.28043210136548 6.61319790816712 6.75776892191451 7.71672845429687 6.46351919711888 6.00222937778313 7.92762166281451 8.06579125488865 8.86642826282840 7.64750633519404 8.19537204739000 8.74829787938987 8.04981225880281 7.44533771135989 7.40378738825686 6.88504039670704 6.89571566393987 8.49894276648130 9.22032075236721 9.51701435052071 10.12654824179192 8.57759897066246 9.44046577239176 9.77158430111749 8.95304837768715
4.57276588623503 2.73814962426146 3.86480088312608 3.52612825912821

3.71621990868884

4.14324852191684 2.47985930539789 3.59230486348452 2.54829974523210 4.20298761633529 3.84312346822034 5.35298786529607 5.64165031137324 5.92726305290671 5.64833236645114 3.26770879036429

2.48951098216549

4.11612842916833 4.99282146040743 5.41860397565650 4.86900277965683

5.69810868354459

3.79922535100855

4.54219907594042

4.17232766999349

2.46692634514756

1.76333562965928

2.59276739477381

0.44717352106933

0.38018756936984

$-0.06049546874661$

$-0.05736120879263$

2.53331983426292

2.93768381184917

1.78194914682869

3.64303108796667

4.01430783967180

4.49115705061347

4.37284820464522

4.90603539936730

4.04913181134281

5.05573693961928

2.33401750151810

1.49906899799871

2.90601334560194

1.78200284747536

2.39163861493475

3.31275070646984

1.86366887479135

0.61243780022613

0.10713638948592

$-0.02244993099185$

0.61366901470178

2.59145970574456

3.89856162228172

4.38335727956593

3.73125260851597

4.59488139945771

1.70504261355265

2.23337634954694

0.78298812471513 
H 6.31350660846858

C 6.91621309070920

H 7.82450328503147

H 6.35328886083541

H 7.21565093320938

C 3.94312083541958

H 4.80275951380181

H 3.04546551182171

C 5.37531763413762

H 5.59443715615567

H 5.28927250826718

H 6.20969756514223

C 4.24116377750340

H 4.63498152441950

H 4.98050695130176

C 2.92723772539271

H 2.67562105389214

H 3.05574084227037

C 0.55695088434826

H 0.65122150690215

H $\quad-0.26103066742807$

H 0.34401501801430

C 1.60730889391943

H 0.61805356485273

H 1.61232041369566

C 2.64360211569682

H 3.62594890576137

H 2.39361915545868

C 4.04288827772200

H 4.16146756878342

H 4.15675271193788

H 4.82720598870331

C 1.68820279161704

H 1.95834610398792

H $\quad 0.75390169183855$

N 2.73521517189179

N 1.79387272758488

N 4.12621677384936

$\mathrm{N} \quad 0.52667376882151$

N -1.83849510610654

N -0.93310078642003

Ni 2.22728421810328

Ni 0.19264831391094

O 3.10454865382361

O 1.91191640866999

O 0.28213763316380

S -0.42507418088088

S 1.20819213002222
10.32367085043759

7.25387079104977

7.54158131251610

6.55567843078395

6.72483286069021

6.35657336979204

6.84246336246750

6.62815009984598

4.56136683872431

3.50324875333355

4.79616747232120

5.13290542795288

4.51788721020682

3.50330248126214

5.15503786239460

4.62404349014595

5.67681821932730

4.21161142034171

4.70544875032086

5.74773990320652

4.26138479879994

4.65125446100086

2.57569416477009

2.28955231242616

2.46021294749798

1.65309482193181

1.85628169741238

0.62006008365752

1.31662856816802

0.24023806539064

1.51705587572831

1.83624916327569

0.93146053387467

$-0.11195861259494$

1.14473363941854

1.78619877779109

3.98770503018549

4.89413043424733

6.87168208877255

5.81857935493896

3.71696342049087

3.92922814767912

4.97518193273363

3.59718243681049

4.27469732262632

3.28816810942239

3.06677109856903

6.00544455190748
1.43353938562776

2.91642385772409

3.45223034320879

3.54122179172850

2.00912065085725

$-1.41631598936060$

$-1.90061247708611$

$-1.97318488170376$

$-0.83262118561697$

$-0.93670227856346$

0.22403550087596

$-1.26197625411998$

$-2.92801453221800$

$-2.96786731736821$

$-3.43581150829572$

$-3.67559199730448$

$-3.80514225302491$

$-4.68490100008310$

$-3.31390826716415$

$-3.01127031008873$

$-2.75318310316322$

$-4.38934437680571$

$-3.35456435193443$

$-2.99656703541579$

$-4.44755205653480$

$-2.75849345872643$

$-3.18459163196323$

$-3.03951496560916$

$-0.84526609819862$

$-1.03066934618317$

0.21709678453913

$-1.39062847431045$

$-0.68700063471072$

$-0.90497873970201$

$-1.19578606413858$

$-1.29713444246397$

$-2.98836153368066$

$-1.51545135172501$

3.20964507996160

1.84382825064747

3.69706088843514

$-0.85713721571459$

1.99234777282473

0.96003053013894

2.73241157521832

$-0.81038215417782$

0.55170067861424

$-0.05782094716302$

Table S10. Calculated bond lengths $/ \AA$ and angles $/{ }^{\circ}$ in $\left[\mathrm{Ni}_{2} \mathrm{~L}^{\mathrm{SO}}\left(\mathrm{O}_{2} \mathrm{CPh}\right)\right]^{+}$(pre-2, sulfenate, PBE0/ def2-TZVP level of theory). ${ }^{\text {a) }}$

\begin{tabular}{|l|l|l|l|l|}
\hline & sulfenate & & & sulfenate \\
\hline Ni1-N1 & 2.246 & & Ni2-N4 & 2.278 \\
\hline Ni1-N2 & 2.176 & & Ni2-N5 & 2.204 \\
\hline Ni1-N3 & 2.229 & & Ni2-N6 & 2.399 \\
\hline Ni1-O1 & 2.045 & & Ni2-O2 & 1.999 \\
\hline Ni1-S2 & 2.447 & & Ni2-S1 & 2.470 \\
\hline Ni1-O3 & 2.049 & & Ni2-S2 & 2.509 \\
\hline S1-O3 & 1.551 & & Ni1 $\cdots$ Ni2 & $\mathbf{3 . 6 5 4}$ \\
\hline
\end{tabular}




\begin{tabular}{|l|l|l|l|l|}
\hline & & & & \\
\hline & & & & \\
\hline N1-Ni1-N2 & 83.0 & & N4-Ni2-N5 & 81.5 \\
\hline N1-Ni1-N3 & 99.4 & & N4-Ni2-N6 & 97.2 \\
\hline N1-Ni1-S2 & 165.4 & & N4-Ni2-S1 & 172.6 \\
\hline N1-Ni1-O1 & 85.5 & & N4-Ni2-O2 & 88.2 \\
\hline N1-Ni1-O3 & 85.4 & & N4-Ni2-S2 & 92.0 \\
\hline N2-Ni1-N3 & 82.5 & & N5-Ni2-N6 & 79.4 \\
\hline N2-Ni1-S2 & 102.4 & & N5-Ni2-S1 & 91.5 \\
\hline N2-Ni1-O1 & 163.8 & & N5-Ni2-O2 & 162.1 \\
\hline N2-Ni1-O3 & 80.9 & & N5-Ni2-S2 & 99.3 \\
\hline N3-Ni1-S2 & 94.8 & & N6-Ni2-S1 & 83.8 \\
\hline N3-Ni1-O1 & 88.1 & & N6-Ni2-O2 & 87.2 \\
\hline N3-Ni1-O3 & 162.0 & & N6-Ni2-S2 & 170.3 \\
\hline S2-Ni1-O1 & 91.5 & & S1-Ni2-O2 & 99.2 \\
\hline S2-Ni1-O3 & 82.1 & & S1-Ni2-S2 & 86.7 \\
\hline O1-Ni1-O3 & 109.6 & & O2-Ni2-S2 & 95.6 \\
\hline
\end{tabular}

a) This structure is more stable than the sulfone form.

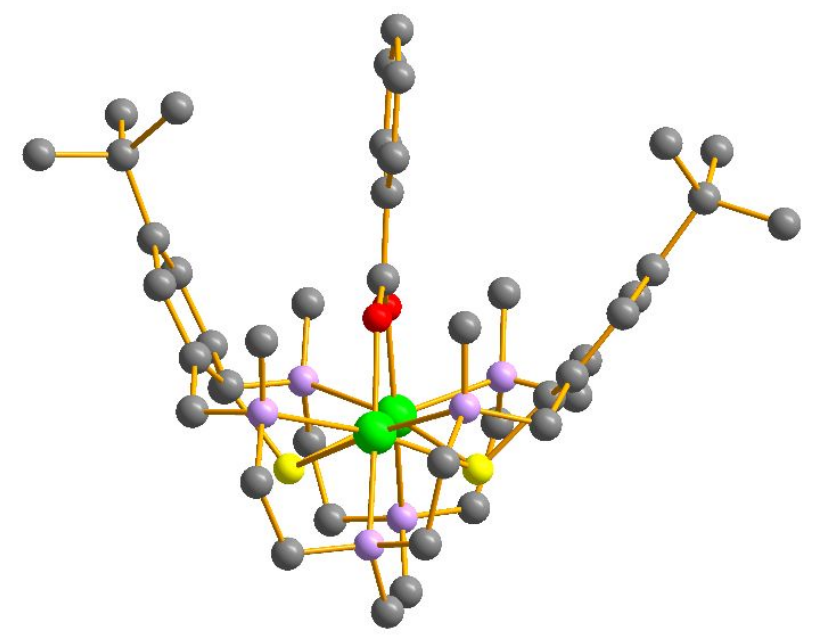

Figure S13. Molecular structure of the $\left[\mathrm{Ni}_{2} \mathrm{~L}^{\mathrm{S}}\left(\mathrm{O}_{2} \mathrm{CPh}\right)\right]^{+}$cation (1) calculated at the PBE0/ def2-TZVP level of theory. Atom labels omitted. Bond lengths and angles are given in the Table below.

Table S11. Comparison of experimental (X-ray) and calculated (gas phase, PBE0/ def2TZVP) bond lengths / $\AA$ and angles $/{ }^{\circ}$ in $\left[\mathrm{Ni}_{2} \mathrm{~L}^{\mathrm{S}}\left(\mathrm{O}_{2} \mathrm{CPh}\right)\right]^{+}(\mathbf{1})$.

\begin{tabular}{|l|l|l|l|l|l|l|}
\hline & $\exp ^{\text {a) }}$ & calc & & & $\exp ^{\text {a) }}$ & calc \\
\hline Ni1-N1 & $2.322(3)$ & 2.345 & & Ni2-N4 & $2.274(3)$ & 2.227 \\
\hline Ni1-N2 & $2.137(3)$ & 2.163 & & Ni2-N5 & $2.133(3)$ & 2.161 \\
\hline Ni1-N3 & $2.197(3)$ & 2.252 & & Ni2-N6 & $2.259(3)$ & 2.379 \\
\hline Ni1-O1 & $2.007(2)$ & 2.002 & & Ni2-O2 & $2.004(2)$ & 1.994 \\
\hline Ni1-S1 & $3.452(1)$ & 2.486 & & Ni2-S1 & $2.506(2)$ & 2.477 \\
\hline Ni1-S2 & $2.509(2)$ & 2.431 & & Ni2-S2 & $2.445(1)$ & 2.443 \\
\hline & & & & & & \\
\hline & & & & & & \\
\hline
\end{tabular}




\begin{tabular}{|l|l|l|l|l|l|l|}
\hline & & & & & & \\
\hline N1-Ni1-N2 & $80.83(12)$ & 80.484 & & N4-Ni2-N5 & $82.29(12)$ & 84.457 \\
\hline N1-Ni1-N3 & $97.49(11)$ & 97.733 & & N4-Ni2-N6 & $97.98(12)$ & 96.717 \\
\hline N1-Ni1-S2 & $170.46(8)$ & 170.391 & & N4-Ni2-S1 & $170.09(9)$ & 172.453 \\
\hline N1-Ni1-O1 & $87.01(10)$ & 89.294 & & N4-Ni2-O2 & $88.15(11)$ & 87.287 \\
\hline N1-Ni1-S1 & $91.41(8)$ & 88.977 & & N4-Ni2-S2 & $90.67(9)$ & 90.071 \\
\hline N2-Ni1-N3 & $83.63(12)$ & 82.680 & & N5-Ni2-N6 & $82.88(12)$ & 79.584 \\
\hline N2-Ni1-S2 & $97.14(9)$ & 95.981 & & N5-Ni2-S1 & $96.08(9)$ & 95.154 \\
\hline N2-Ni1-O1 & $164.57(11)$ & 164.251 & N5-Ni2-O2 & $165.33(11)$ & 164.051 \\
\hline N2-Ni1-S1 & $98.66(10)$ & 96.411 & & N5-Ni2-S2 & $97.85(9)$ & 95.923 \\
\hline N3-Ni1-S1 & $171.06(9)$ & 172.962 & & N6-Ni2-S1 & $91.49(9)$ & 90.614 \\
\hline N3-Ni1-O1 & $88.62(11)$ & 86.901 & & N6-Ni2-O2 & $87.48(11)$ & 87.880 \\
\hline N3-Ni1-S2 & $91.52(8)$ & 90.628 & & N6-Ni2-S2 & $171.33(9)$ & 171.419 \\
\hline S1-Ni1-O1 & $91.05(8)$ & 95.344 & S1-Ni2-O2 & $95.20(8)$ & 94.799 \\
\hline S2-Ni1-O1 & $96.38(8)$ & 95.909 & S1-Ni2-S2 & $79.84(4)$ & 82.466 \\
\hline S1-Ni1-S2 & $79.64(4)$ & 82.514 & & O2-Ni2-S2 & $93.35(8)$ & 97.713 \\
\hline
\end{tabular}

Table S12: Cartesian coordinates / $\AA$ of geometry optimized $\left[\mathrm{Ni}_{2} \mathrm{~L}^{\mathrm{S}}\left(\mathrm{O}_{2} \mathrm{CPh}\right)\right]^{+}(\mathbf{1}$, gas phase, PBE0/ def2-TZVP).

\begin{tabular}{cccc}
\hline atom $\mathrm{x}$ & $\mathrm{y}$ & $\mathrm{z}$ \\
\hline $\mathrm{Ni}$ & 1.82881394659173 & 6.85714083934725 & 0.65531266580810 \\
$\mathrm{~S}$ & 3.51879113721015 & 6.54500011728468 & -1.08062646852852 \\
$\mathrm{~S}$ & 0.28435270382498 & 6.68126930366795 & -1.27371571464044 \\
$\mathrm{O}$ & 1.64180676201567 & 4.94610259086141 & 1.19350705908556 \\
$\mathrm{~N}$ & 3.43314980365326 & 7.02270265248289 & 2.19128229297199 \\
$\mathrm{~N}$ & 1.90039144688210 & 9.01710661083394 & 0.65040625557850 \\
$\mathrm{~N}$ & 0.00645215816947 & 7.35111049368732 & 2.10209885523147 \\
$\mathrm{Ni}$ & 1.93936497324853 & 5.07470130066155 & -2.20125657968704 \\
$\mathrm{C}$ & 4.66760588019758 & 5.42973744233815 & -0.37250383224182 \\
$\mathrm{C}$ & -0.85743275392526 & 5.53906806605490 & -0.58065588007119 \\
$\mathrm{C}$ & 1.69839018104139 & 3.87435960672397 & 0.54527034701534 \\
$\mathrm{C}$ & 4.79552052932650 & 6.88741446224377 & 1.63874273301269 \\
$\mathrm{C}$ & 3.32982454476398 & 6.07125131156140 & 3.29669495575456 \\
$\mathrm{C}$ & 3.25310470539275 & 8.39066671183766 & 2.68264173784067 \\
$\mathrm{C}$ & 2.98838313002106 & 9.39264683608067 & 1.57061288120909 \\
$\mathrm{C}$ & 2.15551201771804 & 9.58508744755619 & -0.67908824208444 \\
$\mathrm{C}$ & 0.58705461786656 & 9.50954142429715 & 1.09146423447949 \\
$\mathrm{C}$ & 0.05925665754384 & 8.79993891576845 & 2.31457883220142 \\
$\mathrm{C}$ & -0.08391594950444 & 6.66627358450698 & 3.38683864896331 \\
$\mathrm{C}$ & -1.23110526119263 & 7.05003435337904 & 1.35365130760577 \\
$\mathrm{O}$ & 1.78273514209064 & 3.75858200705904 & -0.70088265939856 \\
$\mathrm{~N}$ & 0.18862092419336 & 3.97577961778676 & -3.30945139120123 \\
$\mathrm{~N}$ & 2.11081155265076 & 6.00140981824017 & -4.14771192222755 \\
$\mathrm{~N}$ & 3.63129188536331 & 3.80610388480126 & -2.97498746677332 \\
$\mathrm{C}$ & 5.09096770874430 & 5.59619189778188 & 0.94497041353546 \\
$\mathrm{C}$ & 5.19786154418222 & 4.37482404856643 & -1.11785166372933 \\
$\mathrm{C}$ & -1.33348962118327 & 5.70374118880820 & 0.71800144490590 \\
$\mathrm{C}$ & -1.28374534882644 & 4.42886826052398 & -1.31130109846326 \\
$\mathrm{C}$ & 1.67422805927568 & 2.60790324548806 & 1.33981226561042 \\
$\mathrm{H}$ & 5.51106082901514 & 7.02954837253075 & 2.46109645033089 \\
$\mathrm{H}$ & 4.95094764371676 & 7.69312448357810 & 0.91903815367650 \\
$\mathrm{H}$ & 3.51508103497500 & 5.06843581126042 & 2.92158112059280 \\
$\mathrm{H}$ & 2.33442197241175 & 6.08762009376230 & 3.72251360497130 \\
$\mathrm{H}$ & 4.06274330756883 & 6.30991820133395 & 4.07925384631642
\end{tabular}


H 4.14164975591261

H 2.43672282770696

H 2.78799430106241

H 3.89083416198948

H 1.37051553988930

H 3.11481695409257

H 2.15614793882649

H $\quad-0.09578516993362$

H 0.63146514933054

H 0.68389741045995

H $\quad-0.93729756042487$

H -0.01863958508463

H -1.03414391874601

H 0.72048288643856

H $\quad-1.32393847930809$

H $\quad-2.08384546421992$

C -1.10956510672928

C 0.18929330369024

C 0.28583146449905

C 0.80249253767075

C 2.38995872726537

C 3.20216067483433

C 3.47787103192321

C 3.59886708013485

C 4.95694970130884

C 5.87714120064987

C 6.00073495880464

C -2.01081797296147

C -1.96804724611929

C 1.89452002318734

C 1.44208738508641

H -1.90273477499103

H -1.23987349508834

H $\quad 0.25148330543987$

H 1.03623188257727

H $\quad-0.72368044360675$

H $\quad-0.69348474209611$

H 0.93613186137295

H 0.84786627948459

H 0.11303537965963

H 3.32128840282409

H 1.58303902071146

H 2.46346476567628

H 4.10172620578460

H 2.99781102527099

H 2.67567096534241

H 4.38256192974994

H 3.77993862353217

H 4.37111661791883

H 2.62873344230153

H 5.05202301322593

H 5.72650280285746

H 6.16232625411300

C 6.30903002917027

H 6.39272508814448

C -2.27795385774898

H -2.34714557200915

H -2.27388127004763

H 2.05948332385841

C 1.90344889074723

C 1.43637801134547
8.72367684576828

8.37516088248131

10.37495450554590

9.50460709421731

9.25192800196724

9.22984102492509

10.68191627859372

9.36037000202796

10.59006020431310

9.01333840571026

9.19505555933199

5.59061151632862

6.89861136880311

6.98568640733803

7.78832247847025

7.19324305303752

4.45224579910531

2.52097297201682

4.40838390498037

5.82288455868419

7.43890433115885

5.33083251274389

3.90741081833717

2.40195352291683

4.33421526957392

4.61795172673176

3.42813912404173

4.65978752155021

3.40811941409055

1.38467206299826

2.63980756652664

3.85393996532563

5.48394897279329

2.22563789487157

2.10706620593924

2.09807544236839

4.35131068899312

3.72713836235990

6.13784757883969

6.49519159633560

7.61300929150865

7.93812257852062

7.86303534785534

5.92913520494407

5.33799642510093

3.23416673156543

3.54581962479775

2.33283246223753

1.83138709823915

1.96575545666864

5.34788143731316

3.71262068989043

4.76127725681043

3.48479651319071

2.61630010138476

3.46081714646287

4.80239902341610

2.54838285825091

1.36796445968610

0.21213521607528

1.46674622879908
3.23962044645033

3.40134068691551

2.01890797481112

0.96873800576488

$-1.35781856760677$

$-1.05149935589328$

$-0.65027088808056$

0.25385596845010

1.28498918981037

3.18093363446821

2.56039449458875

3.23089844371241

3.88773870672326

4.04562181240131

0.55688335430423

2.03300363783164

$-2.79084139672468$

$-3.21494649833452$

$-4.70637514810994$

$-4.79299154590474$

$-4.09261401651055$

$-4.87768125487335$

$-4.42537826804235$

$-2.56961159203999$

$-2.59042916993973$

1.54388733735108

$-0.49826929559540$

1.33561307213534

$-0.67420733394086$

0.71648012635710

2.71130677645954

$-3.26105432815374$

$-3.11959936698987$

$-2.16821787866851$

$-3.75652096761477$

$-3.65612172636575$

$-5.20120616141705$

$-5.25258787204747$

$-5.84478667640238$

$-4.28032244497885$

$-3.55697200010648$

$-3.55660886673366$

$-5.10193191557906$

$-4.73285127031234$

$-5.95597272649844$

$-4.71975214507610$

$-4.93699858450167$

$-1.50088619967810$

$-3.10418376045753$

$-2.77513866796948$

$-2.98288152971790$

$-3.07016963125231$

2.57943043700942

0.85939803067018

$-1.10203181470560$

0.68428476073722

2.35567809539288

$-1.26158718092802$

$-0.35220433306505$

1.45315305490698

3.44833800301245 


$\begin{array}{llcc}\text { H } & 1.25848382505017 & 3.59439946748750 & 3.18695471351039 \\ \text { C } & 7.12264856037025 & 2.37714345653011 & 1.52221223432014 \\ \text { C } & -2.89336360558438 & 2.25616218605614 & 1.38218983617006 \\ \text { H } & 2.08525450140567 & -0.73547480281924 & 0.96020007592348 \\ \text { C } & 1.67327943496851 & 0.25047014778403 & 2.82143122485978 \\ \text { H } & 1.23919449549186 & 1.49612661738602 & 4.51367350418828 \\ \text { C } & 8.57427726581137 & 2.42736394410407 & 1.03593928869154 \\ \text { C } & 6.54363923682970 & 1.00125452086627 & 1.17529261287408 \\ \text { C } & 7.11229446915591 & 2.50821499705455 & 3.04059399900535 \\ \text { C } & -1.91269076140894 & 1.08422501044028 & 1.27061469644722 \\ \text { C } & -3.13902136999420 & 2.52283664773235 & 2.86125936603941 \\ \text { C } & -4.22731482149646 & 1.88065756798158 & 0.73107394320799 \\ \text { H } & 1.67231503910615 & -0.66655569688061 & 3.39855651485452 \\ \text { H } & 9.02287765404056 & 3.40371930753044 & 1.23252854626010 \\ \text { H } & 8.64549320815799 & 2.22328602130590 & -0.03556433386332 \\ \text { H } & 9.16832357162440 & 1.67155706588124 & 1.55544155103576 \\ \text { H } & 7.09251109547851 & 0.22790871088989 & 1.71759604237669 \\ \text { H } & 6.63756164224575 & 0.77700225620507 & 0.11038428161299 \\ \text { H } & 5.48923636328320 & 0.93012731325076 & 1.45549988505108 \\ \text { H } & 7.60298350216033 & 3.42337172496402 & 3.37902880860968 \\ \text { H } & 7.65589034657698 & 1.67087032305330 & 3.48128084560866 \\ \text { H } & 6.09392627399283 & 2.48976799522948 & 3.43651549877884 \\ \text { H } & -2.31562298598828 & 0.20475685692754 & 1.77943417236542 \\ \text { H } & -0.95534871672192 & 1.33565665835825 & 1.73096092249407 \\ \text { H } & -1.72477242418705 & 0.81380210368278 & 0.22942004279207 \\ \text { H } & -2.21043608478247 & 2.76752062331002 & 3.38281789857222 \\ \text { H } & -3.55718362213655 & 1.62860174782298 & 3.32747311520568 \\ \text { H } & -3.85213591061071 & 3.33521596002644 & 3.01947871956249 \\ \text { H } & -4.94113561945592 & 2.70436733389875 & 0.80408414018732 \\ \text { H } & -4.65905871595106 & 1.01354848306110 & 1.23715286443401 \\ \text { H } & -4.11229118921387 & 1.62127785331987 & -0.32363586827436\end{array}$

Energy $=-6046.0529$ Eh

a) There are two crystallographically independent molecules A and B in the unit cell. The values calculated here utilized the fractional coordinates of the non disordered cation A. 

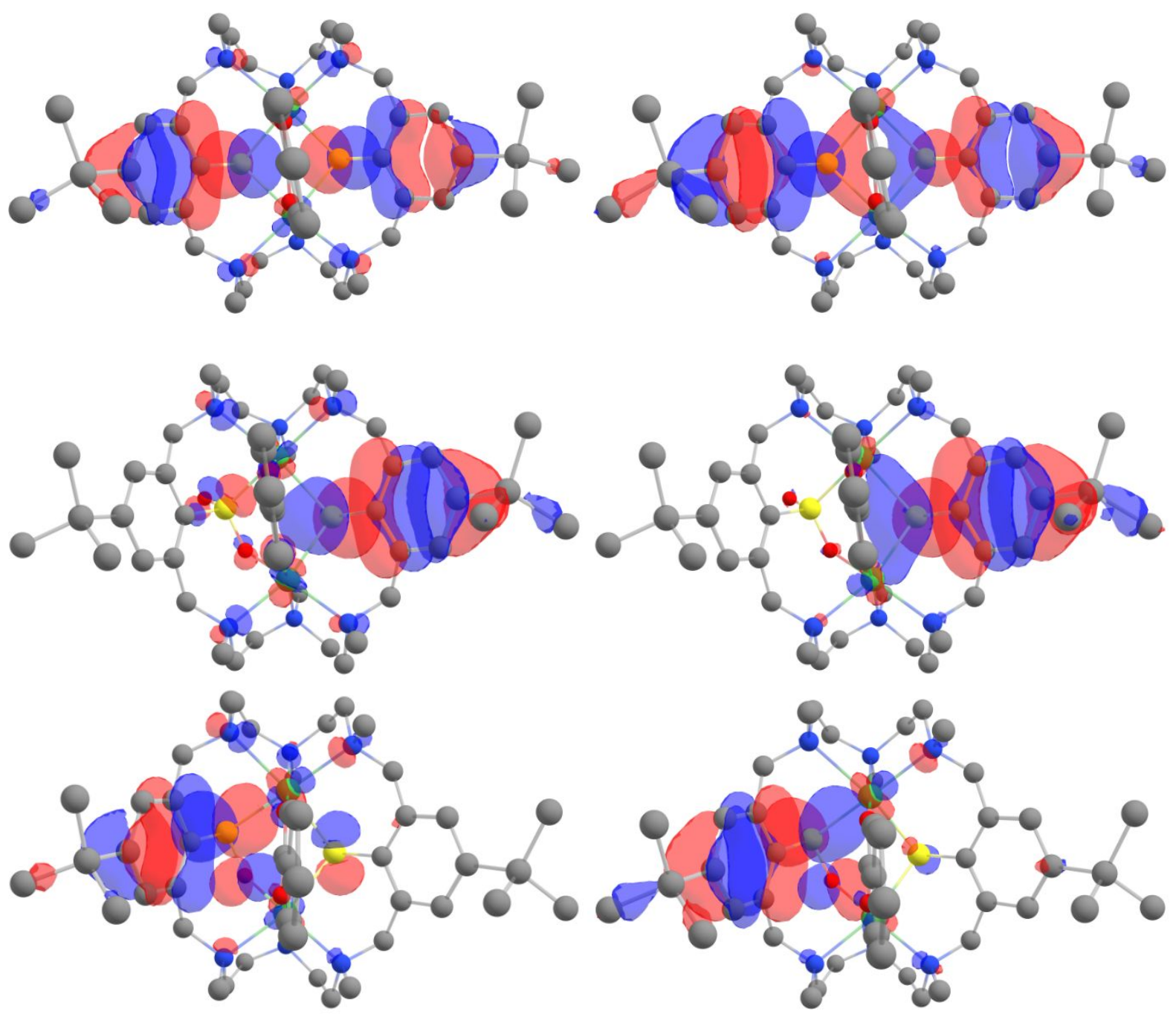

Figure S14. $\alpha$ (left) and $\beta$ HOMO plot (right) of 1 (top), 2 (middle), and pre-2 (sulfenat, bottom) at an Uiso value of 0.03 (B3LYP def2-TZVP def2-TZVP/J, RIJCOSX, gas phase). 
6. Calculation of Electronic Absorption Spectra TD-DFT Calculations

TD-DFT calculations were performed on the nickel complexes 1-3 and the zinc complex $\left[\mathrm{Zn}_{2} \mathrm{~L}^{\mathrm{S}}\left(\mathrm{O}_{2} \mathrm{CPh}\right)\right]^{+}(\mathbf{5})$ in order to shed light on the nature of the absorptions in the 200-500 nm range. The geometry optimized coordinates were taken as input geometry and the 50 lowest excited states were calculated using TD-DFT at the (B3LYP/def2-TZVP) $)^{1}$ level of theory. The energy of the lowest excited state for the zinc complex $\left[\mathrm{Zn}_{2} \mathrm{~L}^{\mathrm{S}}\left(\mathrm{O}_{2} \mathrm{CPh}\right)\right]^{+}(\mathbf{5})$, attributed to a $\pi \rightarrow \pi^{*}$ transition, was calculated to be $299 \mathrm{~nm}\left(f_{\text {osc }}=0.03\right)$. The nickel complexes $\mathbf{1}, \mathbf{2}$ and $\mathbf{3}$ showed intense (f osc > 0.01) transitions at much lower energies (349 nm (1), 389 (2), and $340 \mathrm{~nm}(3))$, respectively, in good agreement with the experimental trend. The 5 lowest exited states for $\mathbf{5}$ are dominated by electron excitations from the HOMO to the LUMO. Inspection of these MOs reveals that their composition is dominated by the $\mathrm{C}, \mathrm{N}$, and $\mathrm{S}$ atoms of the thiophenolate ligands. There are no significant contributions from the $\mathrm{Zn} \mathrm{d}$ orbitals. Thus, our TD-DFT calculations confirm the intraligand origin $\left(\pi-\pi^{*}, \mathrm{n}^{\text {thiophenolato(S) }}-\pi^{*}\right)$ of the absorptions bands that dominate the absorption spectra of the $\mathrm{ZnL}$ complexes in the 300-200 $\mathrm{nm}$ region.

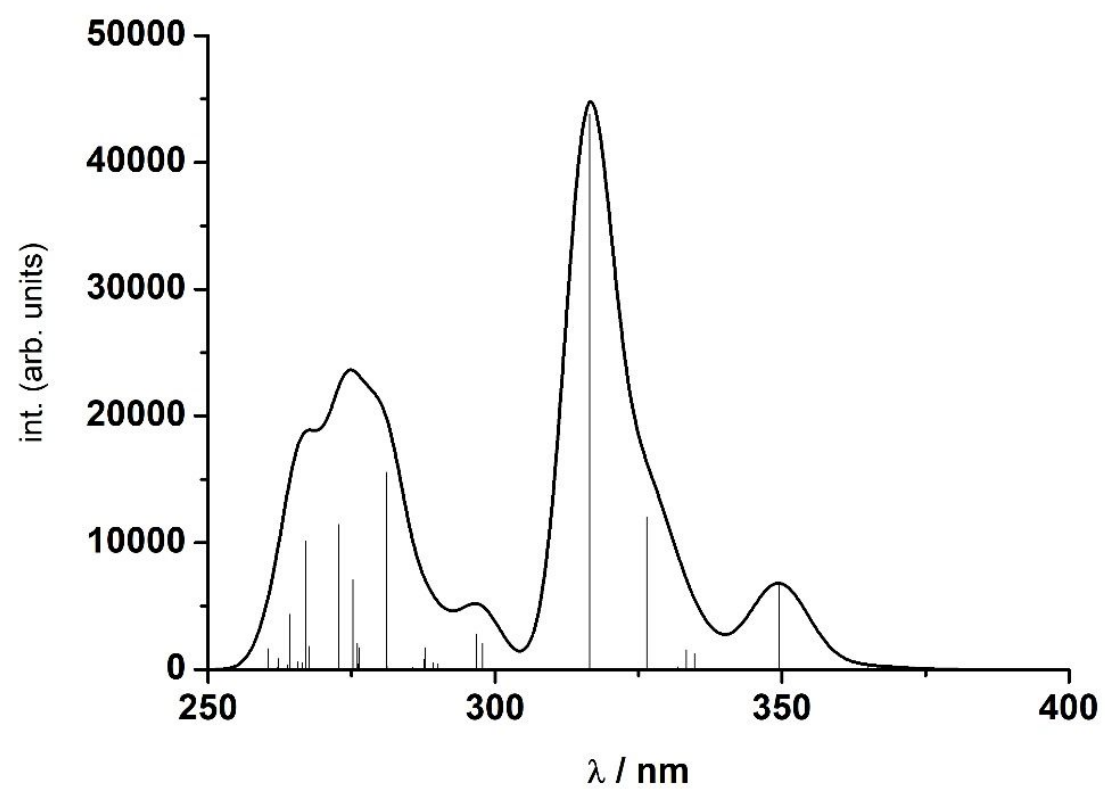

Figure S15. TD-DFT calculated UV-vis spectrum for $\left[\mathrm{Ni}_{2} \mathrm{~L}^{\mathrm{S}}\left(\mathrm{O}_{2} \mathrm{CPh}\right)\right]^{+}(\mathbf{1})$ at the def2TZVP/B3LYP level of theory (gas phase, FWHM $1000 \mathrm{~cm}^{-1}$ ). 
Table S13. TD-DFT calculated transitions $\left(f_{\text {osc }}\right.$ (oscillator strength) $\left.>0.01\right)$ in the region 200 $\mathrm{nm}<\lambda<400 \mathrm{~nm}$ for $\left[\mathrm{Ni}_{2} \mathrm{~L}^{\mathrm{S}}\left(\mathrm{O}_{2} \mathrm{CPh}\right)\right]^{+}$(1) (B3LYP def2-TZVP def2-TZVP/J, RIJCOSX, gas phase) and their assignments. The transitions are depicted as difference densities $\psi_{\mathrm{ES}}-\psi_{\mathrm{GS}}$ at a contour value of 0.006 . The transition proceeds from red to green.

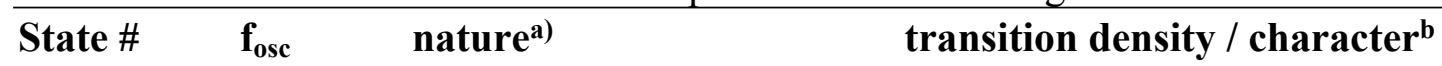
$\# / \lambda[\mathrm{nm}]$

$15 / 349 \quad 0.03 \quad \beta$-HO $\rightarrow \beta$-LU+1 $(90 \%)$

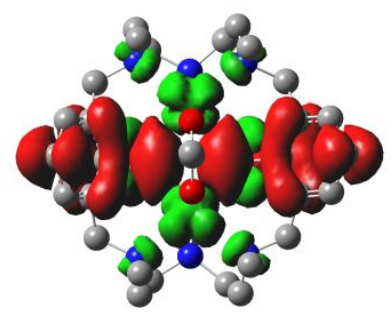

LMCT $(\pi$ (thiolate $) \rightarrow e_{g}$

$20 / 326 \quad 0.06 \quad \alpha-\mathrm{HO} \rightarrow \alpha-\mathrm{LU}(12 \%)$

$\beta$-HO $\rightarrow \beta$-LU+2 (56\%)

$\beta-\mathrm{HO} \rightarrow \beta-\mathrm{LU}+7(56 \%)$

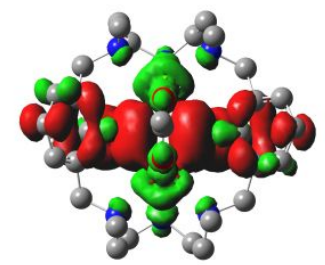

LMCT $\left(\pi\right.$ (thiolate) $\rightarrow e_{g}$ $\left(\mathrm{Ni}^{2+}\right)$

$22 / 316 \quad 0.20 \quad \beta$-HO-2 $\rightarrow \beta$-LU+2 $(10 \%)$

$\beta$-HO- $1 \rightarrow \beta$-LU $(67 \%)$

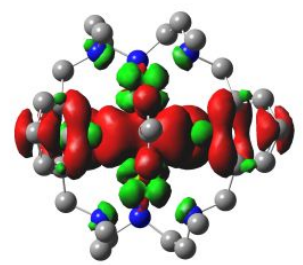

$\operatorname{LMCT}(\pi$ (thiolate $) \rightarrow e_{g}$

$25 / 297 \quad 0.01 \quad \alpha$-HO $\rightarrow \alpha$-LU+3 $(28 \%)$

$\beta$-HO- $1 \rightarrow \beta$-LU+2 (67\%)

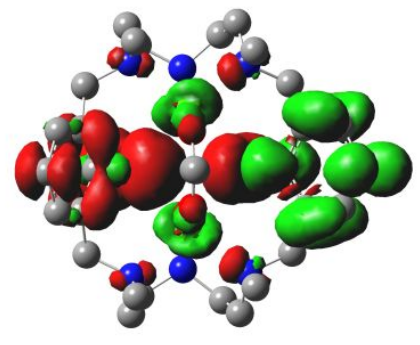

LMCT $(\pi$ (thiolate $) \rightarrow e_{g}\left(\mathrm{Ni}^{2+}\right)$

$+\operatorname{ILCT}(\pi$ (thiolate $) \rightarrow \pi *($ thiolate $))$ 
$34 / 281$

$0.07 \quad \beta$-HO-1 $\rightarrow \beta$-LU+2 $(50 \%)$

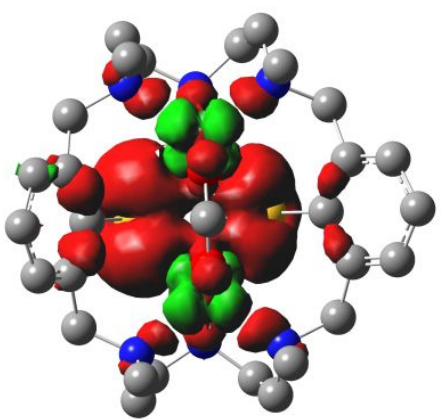

LMCT $\left(p(\right.$ thiolate $\left.) \rightarrow \mathrm{e}_{\mathrm{g}}\left(\mathrm{Ni}^{2+}\right)\right)$

a) Transition contribution $>10 \%$. b) Tert-butyl groups and hydrogen atoms omitted for clarity.

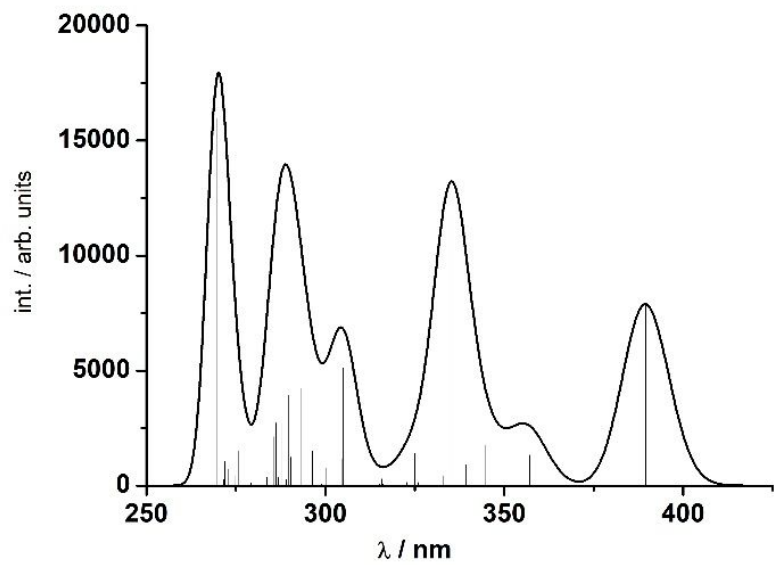

Figure S16. TD-DFT calculated UV-vis spectrum for $\left[\mathrm{Ni}_{2} \mathrm{~L}^{\mathrm{SO} 2}\left(\mathrm{O}_{2} \mathrm{CPh}\right)\right]^{+}(\mathbf{2})$ at the def2TZVP/B3LYP level of theory (gas phase, FWHM $1000 \mathrm{~cm}^{-1}$ ). 
Table S14. TD-DFT calculated transitions $\left(\mathrm{f}_{\mathrm{osc}}\right.$ (oscillator strength) $\left.>0.01\right)$ in the region 250 $\mathrm{nm}<\lambda<450 \mathrm{~nm}$ for $\left[\mathrm{Ni}_{2} \mathrm{~L}^{\mathrm{SO}}\left(\mathrm{O}_{2} \mathrm{CPh}\right)\right]^{+}$(2) (B3LYP def2-TZVP def2-TZVP/J, RIJCOSX, gas phase) and their assignments. The transitions are depicted as difference densities $\psi_{\mathrm{ES}}-\psi_{\mathrm{GS}}$ at a contour value of 0.006 . The transition proceeds from red to green.

\begin{tabular}{|c|c|c|c|}
\hline $\begin{array}{l}\text { State } \\
\# / \lambda[\mathrm{nm}]\end{array}$ & $\mathrm{f}_{\mathrm{osc}}$ & $\begin{array}{l}\text { orbitals involved } \\
\text { in transition }\end{array}$ & transition density / character $\left.{ }^{b}\right)$ \\
\hline 13 / 389 & 0.04 & $\beta$-HO $\rightarrow \beta-\mathrm{LU}(78 \%)$ & $\begin{array}{l}\mathrm{LMCT}(\pi \text { (thiolate }) \rightarrow e_{g} \\
\left(\mathrm{Ni}^{2+}\right)\end{array}$ \\
\hline 14 / 357 & 0.006 & $\begin{array}{l}\alpha \text {-HO-1 } \rightarrow \alpha \text {-LU }(19 \%) \\
\alpha \text {-HO } \rightarrow \alpha \text {-LU }(35 \%) \\
\beta \text {-HO } \rightarrow \beta \text {-LU+1 }(16 \%)\end{array}$ & $\begin{array}{l}\operatorname{LMCT}(\pi \text { (thiolate }) \rightarrow e_{g}\left(\mathrm{Ni}^{2+}\right) \\
\operatorname{LLCT}(\pi \text { (thiolate }) \rightarrow \pi *(\text { sulfinate })\end{array}$ \\
\hline
\end{tabular}

$15 / 355 \quad 0.006 \quad \alpha$-HO-1 $\rightarrow \alpha$-LU (12\%)

$\alpha$-HO $\rightarrow \alpha$-LU (20\%)

$\beta$-HO $\rightarrow \beta$-LU+1 $(28 \%)$

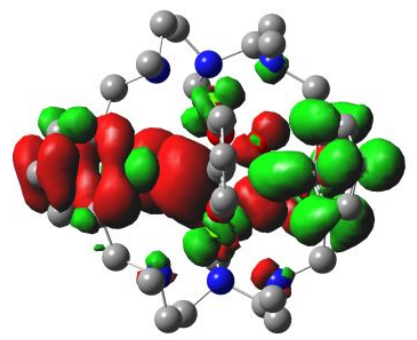

LMCT $(\pi$ (thiolate $) \rightarrow e_{g}\left(\mathrm{Ni}^{2+}\right)$

LLCT $(\pi$ (thiolate $) \rightarrow \pi *$ (sulfinate $)$

$18 / 335 \quad 0.05 \quad \alpha-\mathrm{HO} \rightarrow \alpha$-LU+3 $(20 \%)$

$\beta$-HO $\rightarrow \beta$-LU+2 (20\%)

$\beta$-HO $\rightarrow \beta$-LU+2 (28\%)

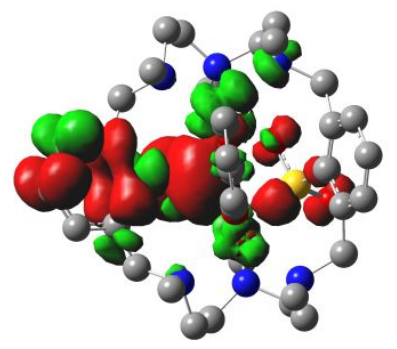

LMCT $(\pi$ (thiolate $) \rightarrow e_{g}\left(\mathrm{Ni}^{2+}\right)$

$\operatorname{LMCT}\left(p(\mathrm{~S}\right.$, sulfinate $\left.) \rightarrow e_{g}\left(\mathrm{Ni}^{2+}\right)\right)$ 


$$
\begin{aligned}
0.02 & \beta \text {-HO-3 } \rightarrow \beta \text {-LU }(20 \%) \\
& \beta \text {-HO-2 } \rightarrow \beta \text {-LU+1 (20\%) } \\
& \beta \text {-HO- } 2 \rightarrow \beta \text {-LU+2 }(28 \%)
\end{aligned}
$$

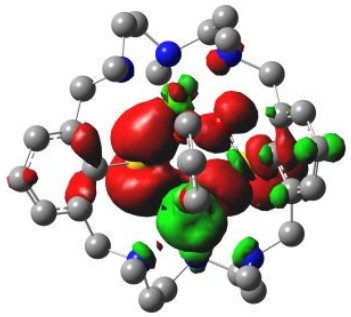

LMCT $\left(p\right.$ (thiolate) $\rightarrow e_{g}\left(\mathrm{Ni}^{2+}\right)$

$\operatorname{LMCT}\left(\pi(\mathrm{S}\right.$, sulfinate $\left.) \rightarrow e_{g}\left(\mathrm{Ni}^{2+}\right)\right)$

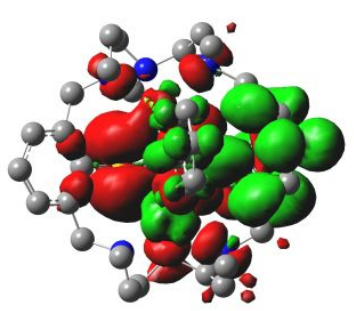

LMCT $\left(p\right.$ (thiolate) $\rightarrow e_{g}\left(\mathrm{Ni}^{2+}\right)$

LLCT $\left(p\right.$ (thiolate) $\rightarrow \pi^{*}($ sulfinate $\left.)\right)$

$34 / 290$

$$
0.02
$$$$
\beta \text {-HO-1 } \rightarrow \beta \text {-LU+2 }(34 \%)
$$$$
\beta \text {-HO-1 } \rightarrow \beta \text {-LU+3 (13\%) }
$$

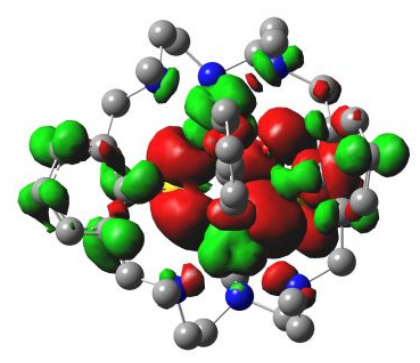

$\operatorname{ILCT}(\pi$ (thiolate $) \rightarrow \pi^{*}($ sulfinate $\left.)\right)$ ILCT ( $\pi$ (sulfinate) $\rightarrow \pi^{*}$ (sulfinate) $)$

\footnotetext{
a) transition contribution $>10 \%$. ${ }^{\text {b) }}$ Hydrogen atoms and tert-butyl groups omitted for clarity.
} 


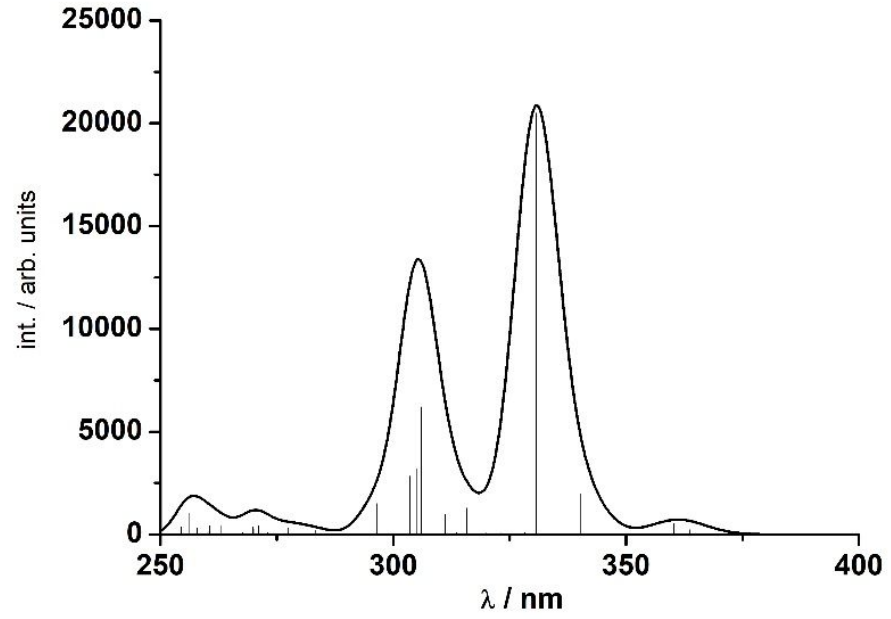

Figure S17. TD-DFT calculated UV-vis spectrum for $\left[\mathrm{Ni}_{2} \mathrm{~L}^{\mathrm{SO}}\left(\mathrm{O}_{2} \mathrm{CPh}\right)\right]^{+}(3)$ at the def2TZVP/B3LYP level of theory (gas phase, FWHM $1000 \mathrm{~cm}^{-1}$ ). 
Table S15. TD-DFT calculated transitions ( $f_{\text {osc }}$ (oscillator strength) $\left.>0.01\right)$ in the region 250 $\mathrm{nm}<\lambda<400 \mathrm{~nm}$ for $\left[\mathrm{Ni}_{2} \mathrm{~L}^{\mathrm{SO} 4}\left(\mathrm{O}_{2} \mathrm{CPh}\right)\right]^{+}$(3) (B3LYP def2-TZVP def2-TZVP/J, RIJCOSX, gas phase) and their assignments. The transitions are depicted as difference densities $\psi_{\mathrm{ES}}-\psi_{\mathrm{GS}}$ at a contour value of 0.006 . The transitions proceed from red to green.
State \#
nature $^{\text {a) }}$
transition density / characterb)

$\# / \lambda[\mathrm{nm}]$

$\begin{array}{lll}15 / 340 \quad 0.01 & \beta-\mathrm{HO} \rightarrow \beta-\mathrm{LU}(60 \%) \\ & \beta-\mathrm{HO} \rightarrow \beta-\mathrm{LU}+1(28 \%)\end{array}$

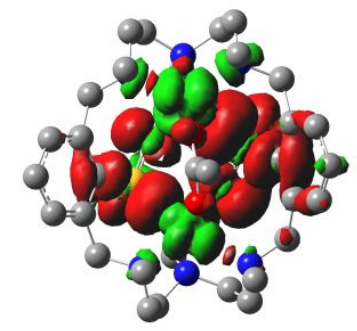

LMCT $\left(\mathrm{pS}\right.$ (sulfinate) $\rightarrow e_{\mathrm{g}}$ $\mathrm{Ni}^{2+}+\mathrm{LMCT}$

$16 / 331$

0.1

$\beta$-HO $\rightarrow \beta$-LU (23 \%)

$\beta-\mathrm{HO} \rightarrow \beta-\mathrm{LU}+1(62 \%)$

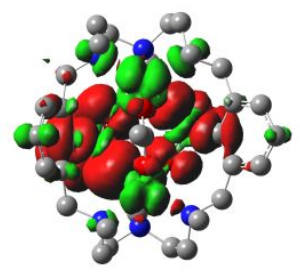

LMCT (pS(sulfinate) $\rightarrow e_{\mathrm{g}}$

$\mathrm{Ni}^{2+}+\mathrm{LMCT}$

$\left(\pi\right.$ (sulfinate) $\rightarrow e_{\mathrm{g}} \mathrm{Ni}^{2+}$

$25 / 306$

0.20

$\alpha$-HO-1 $\rightarrow \alpha-\mathrm{LU}+2(10 \%)$

$\alpha-\mathrm{HO} \rightarrow \alpha-\mathrm{LU}+3(14 \%)$

$\beta-\mathrm{HO}-1 \rightarrow \beta-\mathrm{LU}+1(33 \%)$

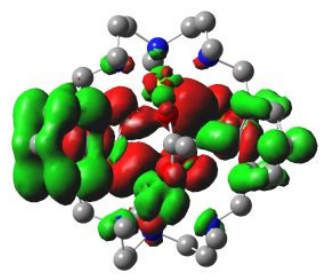

LMCT $\left(\mathrm{pS}(\right.$ sulfinate $) \rightarrow e_{\mathrm{g}} \mathrm{Ni}^{2}$ + II $C$ T

$26 / 306 \quad 0.01 \quad \alpha-H O-1 \rightarrow \alpha-L U+1(15 \%)$

$\alpha-\mathrm{HO} \rightarrow \alpha-\mathrm{LU}+3(24 \%)$

$\beta$-HO- $1 \rightarrow \beta-\mathrm{LU}+1$ (21\%)

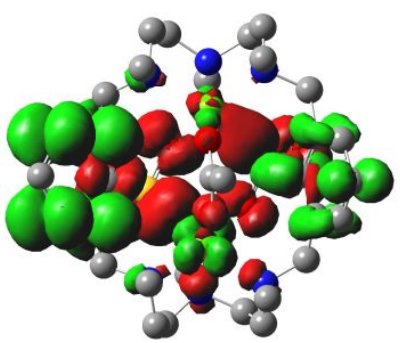

LMCT $\left(\mathrm{pS}(\right.$ sulfinate $) \rightarrow e_{\mathrm{g}} \mathrm{Ni}^{2}$

+ ILCT

$\left(\mathrm{pS}\right.$ (sulfinate) $\rightarrow \pi^{*}$ (sulfinate) 
$27 / 305$

$$
\begin{aligned}
0.07 & \alpha-\mathrm{HO}-1 \rightarrow \alpha-\mathrm{LU}+1(17 \%) \\
& \beta-\mathrm{HO}-1 \rightarrow \beta-\mathrm{LU}+1(12 \%) \\
& \beta-\mathrm{HO}-1 \rightarrow \beta-\mathrm{LU}+3(14 \%) \\
& \beta-\mathrm{HO} \rightarrow \alpha-\mathrm{LU}+5(24 \%)
\end{aligned}
$$

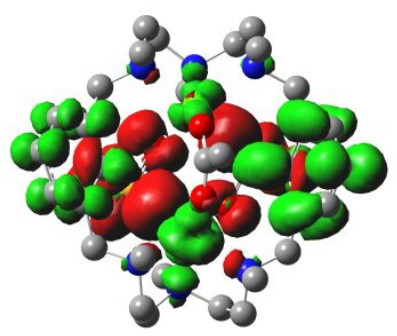

LMCT $\left(\mathrm{pS}(\right.$ sulfinate $) \rightarrow e_{\mathrm{g}} \mathrm{Ni}^{2}$ + ILCT

a) transition contribution $>10 \%$. ${ }^{\text {b) }}$ Hydrogen atoms, tert-butyl groups and parts of co-ligand omitted for clarity.

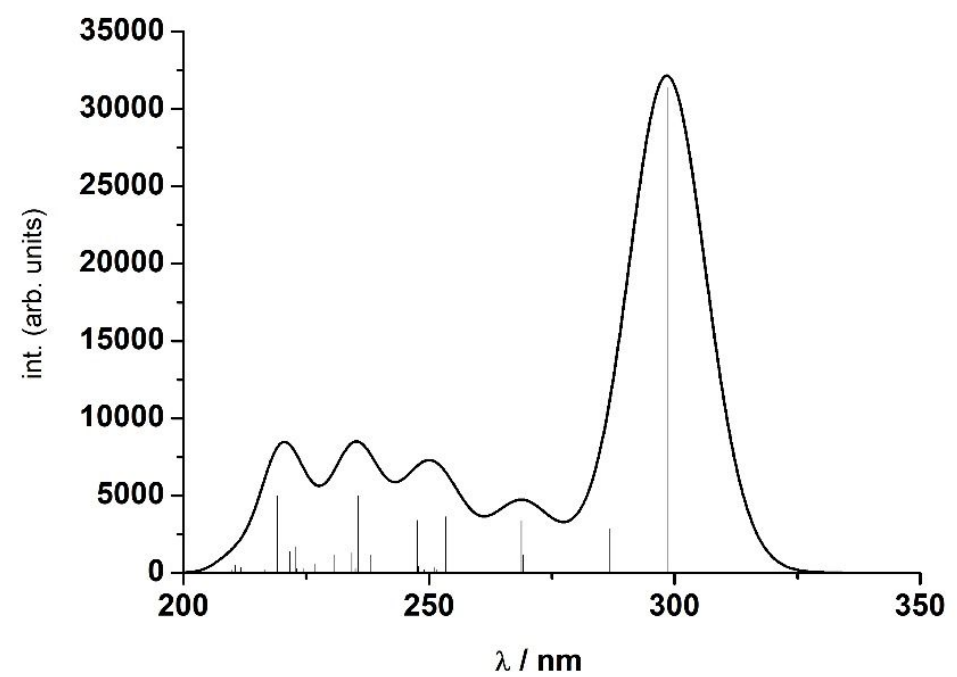

Figure S18. TD-DFT calculated UV-vis spectrum for $\left[\mathrm{Zn}_{2} \mathrm{~L}^{(\mathrm{SAr}) 2}\left(\mathrm{O}_{2} \mathrm{CPh}\right)\right]^{+}(\mathbf{5})$ at the def2TZVP/B3LYP level of theory (gas phase, FWHM $2000 \mathrm{~cm}^{-1}$ ).

Table S16. TD-DFT calculated transitions $\left(f_{\text {osc }}\right.$ (oscillator strength) $\left.>0.01\right)$ and assignments for $\left[\mathrm{Zn}_{2} \mathrm{~L}^{\mathrm{S}}\left(\mathrm{O}_{2} \mathrm{CPh}\right)\right]^{+}$(5) (B3LYP/def2-TZVP, def2-TZVP/J, RIJCOSX, gas phase). The transitions are depicted as difference densities $\psi_{\mathrm{ES}}-\psi_{\mathrm{GS}}$ at a contour value of 0.006 (hydrogen atoms omitted for clarity). The transition proceeds from red to green.
State \# $\mathbf{f}_{\text {osc }}$
nature $^{\text {a) }}$
transition density / character ${ }^{b)}$

$\# / \lambda[\mathrm{nm}]$

$1 / 299 \quad 0.29 \quad \mathrm{HO} \rightarrow \mathrm{LU}(98 \%)$

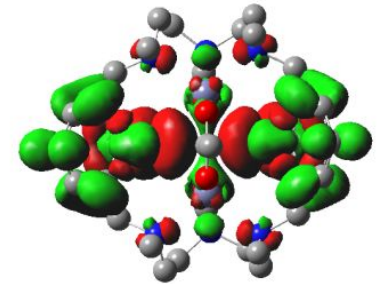

$\operatorname{ILCT}\left(\mathrm{S}->\pi^{*}\right)$ 
$3 / 287 \quad 0.03 \quad \mathrm{HO} \rightarrow \mathrm{LU}+2(86 \%)$

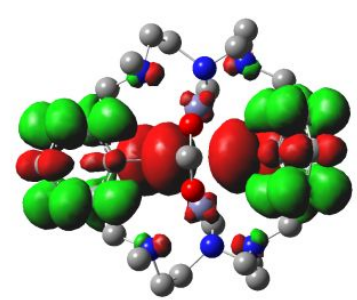

$\pi-\pi^{*}$

$5 / 269 \quad 0.03 \quad$ HO-2 $\rightarrow$ LU (26\%)

$\mathrm{HO} \rightarrow \mathrm{LU}+2(65 \%)$

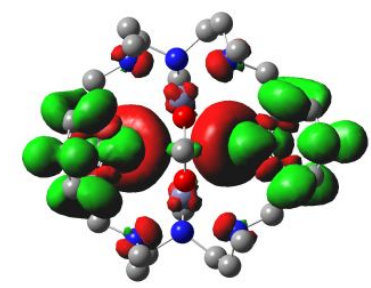

$\operatorname{ILCT}\left(\mathrm{S}->\pi^{*}\right), \pi-\pi^{*}$

$6 / 253 \quad 0.03 \quad$ HO-2 $\rightarrow$ LU+2 (21\%)

$\mathrm{HO}-1 \rightarrow \mathrm{LU}(68 \%)$

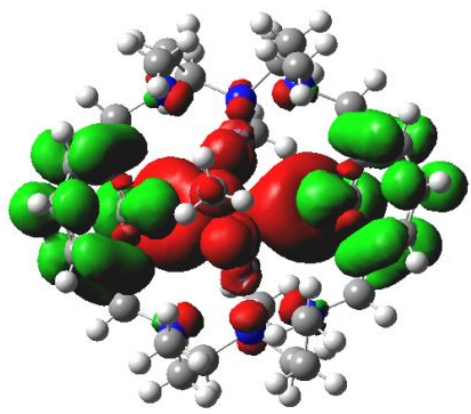

$\operatorname{ILCT}\left(\mathrm{S}->\pi^{*}\right), \pi-\pi^{*}$

$11 / 248 \quad 0.03 \quad \mathrm{HO}-2 \rightarrow \mathrm{LU}+2(65 \%)$

$\mathrm{HO}-2 \rightarrow \mathrm{LU}(15 \%)$

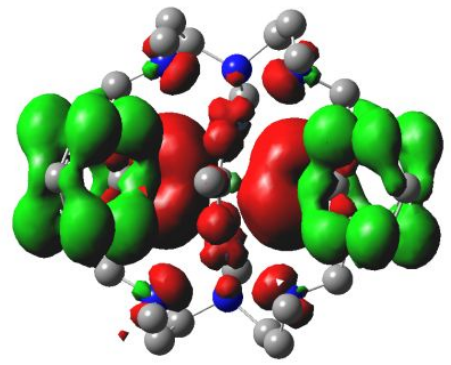

$\operatorname{ILCT}\left(\mathrm{S}->\pi^{*}\right), \pi-\pi^{*}$ 
a) transition contribution $>10 \%$. ${ }^{\text {b) }}$ Hydrogen atoms, tert-butyl groups and parts of co-ligand omitted for clarity.

1 F. Neese, The ORCA program system, WIREs Comput. Mol. Sci., 2012, 2, 73-78. 